\title{
Automorphism Sheaves, Spectral Covers, and the Kostant and Steinberg Sections
}

\author{
Robert Friedman* and John W. Morgan ${ }^{\dagger}$
}

November 1, 2018

\section{Introduction}

Throughout this paper, $G$ denotes a simple and simply connected algebraic group over $\mathbb{C}$ of rank $r$ and $H$ is a Cartan subgroup, with Lie algebras $\mathfrak{g}=\operatorname{Lie} G$ and $\mathfrak{h}=$ Lie $H$. Let $R$ be the root system of the pair $(G, H), W$ the Weyl group, and $\Lambda \subseteq \mathfrak{h}$ the coroot lattice. Fix once and for all a positive Weyl chamber, i.e. a set of simple roots $\Delta$. The geometric invariant theory quotient of $\mathfrak{g}$ by the adjoint action of $G$ is identified with $\mathfrak{h} / W$, which by a theorem of Chevalley is isomorphic to $\mathbb{A}^{r}$. Let $\mathfrak{g}_{\text {reg }} \subseteq \mathfrak{g}$ be the Zariski open and dense subset of $\mathfrak{g}$ consisting of regular elements, i.e. of elements $X$ whose centralizer (in $\mathfrak{g}$ or $G$ ) has the minimal dimension $r$. If $X$ is regular, then the centralizers of $X$ in $\mathfrak{g}$ and in $G$, i.e. the stabilizer of $X$ in $G$ under the adjoint representation, are abelian. One can show that each fiber of the adjoint quotient morphism $\mathfrak{g} \rightarrow \mathfrak{h} / W$ contains a unique orbit of regular elements, so that the restriction of the adjoint quotient morphism to $\mathfrak{g}_{\text {reg }}$ induces an isomorphism $\mathfrak{g}_{\mathrm{reg}} / G \rightarrow \mathfrak{h} / W$. In 1963, Kostant [14] proved that the morphism $\mathfrak{g}_{\mathrm{reg}} \rightarrow \mathfrak{h} / W$ is smooth and constructed a section $\sigma: \mathfrak{h} / W \rightarrow \mathfrak{g}_{\text {reg }}$ of the adjoint quotient morphism, generalizing the existence of rational canonical forms of a matrix. Actually, the construction yields a family of such sections, defined as follows. Let $X \in \mathfrak{g}_{\text {reg }}$ be a principal nilpotent matrix and let $L \subseteq \mathfrak{g}$ be a linear complement to $\operatorname{Im} \operatorname{ad} X$. Then the affine subvariety $L+X$ is contained in $\mathfrak{g}_{\text {reg }}$ and maps isomorphically to $\mathfrak{h} / W$, and so defines a section of the adjoint quotient morphism.

One can make an analogous construction for the adjoint quotient of $G$, i.e. the geometric invariant theory quotient of the action of $G$ on itself by conjugation. In this case, the adjoint quotient is equal to $H / W$, which is again isomorphic to $\mathbb{A}^{r}$ [2]. Let $G_{\text {reg }}$ be the Zariski open and dense subset of regular elements, where by definition $g \in G$ is regular if and only if the dimension of its centralizer is $r$, the minimal possible dimension. If $g$ is regular, then its centralizer is abelian. Then again each fiber of the morphism $G \rightarrow H / W$ contains a unique conjugacy class of regular elements, so that $G_{\text {reg }} / G$ is isomorphic to $H / W$. In 1965 , using

${ }^{*}$ The first author was partially supported by NSF grant DMS-02-00810.

${ }^{\dagger}$ The second author was partially supported by NSF grant DMS-01-03877. 
methods very different from those of Kostant, Steinberg [15] proved that the morphism $G_{\text {reg }} \rightarrow H / W$ is smooth and constructed a section $\sigma: H / W \rightarrow G_{\text {reg }}$ of the adjoint quotient morphism. Although the constructions are quite different, they are related as follows. Let $\sigma: H / W \rightarrow G_{\text {reg }}$ be any section of the adjoint quotient morphism, and let $X \in \mathfrak{g}_{\text {reg }}$ be the unique principal nilpotent matrix such that $\exp X=\sigma(e)$, where we denote by $e$ the image of the identity in $H / W$. Then $\operatorname{Im} d \sigma_{e}$ is a linear subspace $L$ of $\mathfrak{g}$ which is a complement to $\operatorname{Im} \operatorname{ad} X$, and so $L+X$ defines a Kostant section of the adjoint quotient morphism for $\mathfrak{g}$.

In [9], the authors gave another method for constructing sections of the adjoint quotient morphism, either for $\mathfrak{g}$ or for $G$, as well as generalizations to other contexts, which will be explained below. It is natural, then, to ask if these sections are essentially the same as those defined by Kostant and Steinberg, and more generally whether the sections of Kostant and Steinberg are in some sense unique. It follows from the work of Kostant and Steinberg that any such section must take values in the open set of regular elements. Clearly, if $\sigma: \mathfrak{h} / W \rightarrow \mathfrak{g}_{\text {reg }}$ is such a section and $f: \mathfrak{h} / W \rightarrow G$ is any morphism, then ad $f(\sigma): \mathfrak{h} / W \rightarrow \mathfrak{g}_{\text {reg }}$ is again a section, and similarly for sections of $G \rightarrow H / W$. We shall refer to two sections which are related in this way as conjugate. The main result of this paper is then the following:

Theorem. All sections of the adjoint quotient morphism $\mathfrak{g} \rightarrow \mathfrak{h} / W$ are conjugate. Similarly, all sections of the adjoint quotient morphism $G \rightarrow H / W$ are conjugate.

These problems can be translated into a more geometric context. Let $E$ be an irreducible plane cubic curve with a cusp singularity and a marked point $p_{0} \in E_{\text {reg. }}$. Then there is a natural group structure on $E_{\text {reg }}$ with origin $p_{0}$, and it is isomorphic to the additive group $\mathbb{G}_{a}$. Let $n: \widetilde{E} \cong \mathbb{P}^{1} \rightarrow E$ be the normalization of $E$. Let $(\xi, \theta)$ be a pair consisting of a principal $G$-bundle on $E$ and an isomorphism of principal bundles $\theta: n^{*} \xi \rightarrow \widetilde{E} \times G$. By [9, Corollary 3.1.2], such pairs are classified by elements $X \in \mathfrak{g}$, and hence principal $G$-bundles $\xi$ such that $n^{*} \xi$ is trivial are classified by $G$-orbits in $\mathfrak{g}$. If the bundle $\xi$ corresponds to the $G$-orbit containing $X$, then the group Aut $\xi$ of automorphisms of the principal bundle $\xi$ is isomorphic to the stabilizer of $X$ in $G$. Thus, if $\xi$ is a $G$-bundle over $E$ such that $n^{*} \xi$ is trivial, we define $\xi$ to be regular if Aut $\xi$ has dimension $r$. More generally, let $B$ be a scheme over $\mathbb{C}$ and let $(\Xi, \Theta)$ consist of a principal $G$-bundle over $B \times E$ and a bundle isomorphism $\Theta:(\operatorname{Id} \times n)^{*} \Xi \cong B \times \widetilde{E} \times G$. Then such pairs are classified by morphisms $\varphi: B \rightarrow \mathfrak{g}$. Given two such pairs $(\Xi, \Theta)$ and $\left(\Xi^{\prime}, \Theta^{\prime}\right)$ corresponding to morphisms $\varphi$ and $\varphi^{\prime}$ from $B$ to $\mathfrak{g}$, the bundles $\Xi$ and $\Xi^{\prime}$ are isomorphic if and only if there exists a morphism $f: B \rightarrow G$ such that ad $f(\varphi)=\varphi^{\prime}$. Applying this construction in particular to a Kostant section $\sigma: \mathfrak{h} / W \rightarrow \mathfrak{g}_{\text {reg }}$ defines a $G$-bundle $\Xi \rightarrow(\mathfrak{h} / W) \times E$ whose restriction to each slice $\{x\} \times E$ is regular. Clearly, $\mathfrak{h} / W$ is a coarse moduli space for the set of bundles which become trivial on $\widetilde{E}, \Xi$ is a "universal" bundle in an appropriate sense, and our theorem becomes the statement that there is a unique such bundle up to isomorphism.

There is an analogous construction in the case of the group $G$. In this case, let $E$ be an irreducible plane curve with a node and a marked point $p_{0} \in E_{\text {reg. }}$ Then the natural group structure on $E_{\text {reg }}$ with origin $p_{0}$ is isomorphic to $\mathbb{G}_{m}$. Pairs $(\xi, \theta)$ consisting of a principal 
$G$-bundle on $E$ and an isomorphism of principal bundles $\theta: n^{*} \xi \rightarrow \widetilde{E} \times G$ are classified by an element $g \in G$, and more generally pairs $(\Xi, \Theta)$ consisting of a principal $G$-bundle over $B \times E$ and a bundle isomorphism $\Theta:(\operatorname{Id} \times n)^{*} \Xi \cong B \times \widetilde{E} \times G$ are classified by morphisms $\varphi: B \rightarrow G$. Regularity is defined as before. Using the Steinberg section now to define a $G$-bundle over $(H / W) \times E, H / W$ is a coarse moduli space for the set of bundles which become trivial on $\widetilde{E}, \Xi$ is a "universal" bundle in an appropriate sense, and our theorem is again the statement that there is a unique such bundle up to isomorphism.

These constructions can be further generalized. Aside from nodal and cuspidal cubics, one can also consider smooth cubic curves $E$ together with an origin $p_{0}$, i.e. elliptic curves. The group law on $E$ gives it the structure of a one-dimensional algebraic group, analogous to $\mathbb{G}_{a}$ and $\mathbb{G}_{m}$. By analogy with $\mathfrak{h} / W=\left(\mathbb{G}_{a} \otimes \Lambda\right) / W$ and $H / W=\left(\mathbb{G}_{m} \otimes \Lambda\right) / W$, one can also consider $(E \otimes \Lambda) / W=\mathcal{M}_{G}(E)$. By a theorem of Looijenga, $\mathcal{M}_{G}(E)$ is the coarse moduli space of semistable $G$-bundles over $E$, and has been extensively studied in [7, 8] via the so-called parabolic construction. The parabolic construction extends to the case of a nodal or cuspidal curve and defines a compactification of the adjoint quotient of $G$ or $\mathfrak{g}$. Over the adjoint quotient itself, the parabolic construction defines a universal bundle, and this is the method used in [9] to define a section of the adjoint quotient morphism.

For a smooth elliptic curve $E$, one can try to use the methods of this paper to investigate different universal bundles $\Xi$ over $\mathcal{M}_{G}(E) \times E$. However, there are some important differences. The bundle $\Xi$ might only exist as a conformal bundle over $\mathcal{M}_{G}(E) \times E$, i.e. as a bundle over some quotient of $G$ by a subgroup of the center. If $G$ is not of type $A_{n}$ or $C_{n}$, then $\mathcal{M}_{G}(E)$ is singular, and the bundle $\Xi$ will not exist, even locally or in some conformal form of $G$, over the singular points. Finally, even when $\mathcal{M}_{G}(E)$ is smooth, the bundle $\Xi$ will not be unique. However, the methods of this paper, in a relative setting, can be used in many cases to study families of regular semistable bundles over $E$ or over an elliptic fibration.

The general method for proving our main theorem is as follows. We work entirely in the context of $G$-bundles. Let $E$ be a Weierstrass cubic, i.e. a reduced irreducible plane cubic curve with a marked smooth point $p_{0}$. Define $\widetilde{\mathcal{M}}=E_{\text {reg }} \otimes \Lambda$ and $\mathcal{M}=\widetilde{\mathcal{M}} / W$. Given a universal bundle $\Xi \rightarrow \mathcal{M} \times E$, there is a corresponding automorphism sheaf $\mathcal{A}$ over $\mathcal{M}$, defined by $\mathcal{A}(U)=\operatorname{Aut}(\Xi \mid U \times E)$. One can show that, up to isomorphism, $\mathcal{A}$ is independent of the choice of $\Xi$. A standard argument shows that the set of all universal bundles is a principal homogeneous space over $H^{1}(\mathcal{M} ; \mathcal{A})$. Thus we must show that $H^{1}(\mathcal{M} ; \mathcal{A})$ is trivial. This is done by analyzing the sheaf $\mathcal{A}$ and relating it to various finite covers of $\mathcal{M}$ associated to $\Xi$ as follows. Let $\rho: G \rightarrow G L(V)$ be a representation of $G$, which in practice will always be minuscule or quasi-minuscule (to be defined in Section 1). We can then form the associated vector bundle $\mathcal{V}=\Xi \times_{G} V$, which is a family of semistable vector bundles over $\mathcal{M} \times E$. The Fourier-Mukai correspondence functorially associates to $\mathcal{V}$ a finite cover $T$ of $\mathcal{M}$, not necessarily reduced, and a sheaf of $\mathcal{O}_{T}$-modules $\mathcal{Q}$, finite and flat over $\mathcal{M}$, which determines $\mathcal{V}$. The scheme $T$ is called the spectral cover of $\mathcal{M}$ associated to $\mathcal{V}$, and we are able to describe $\mathcal{A}$ in terms of the sheaf of invertible functions on $T$ and various correspondences associated to $T$. Once we have this description, we are able to prove the 
desired vanishing for $H^{1}(\mathcal{M} ; \mathcal{A})$.

Automorphism sheaves and spectral covers have been studied by many authors, including Donagi [1], Donagi-Gaitsgory [5], and Kanev [12]. Knop has shown (cf. [13]) that, if $\pi: \widetilde{\mathcal{M}} \rightarrow \mathcal{M}$ is the projection and $\underline{H}$ is the sheaf over $\widetilde{\mathcal{M}}$ associated to the constant group scheme $\widetilde{\mathcal{M}} \times H$, then $\mathcal{A}=\left(\pi_{*} \underline{H}\right)^{\underline{W}}=\left(\pi_{*} \mathbb{G}_{m, \widetilde{\mathcal{M}}} \otimes \Lambda\right)^{W}$. It is likely that this description could also lead to a proof of the main theorem.

\section{Notation and conventions}

All schemes in this paper will be of finite type over $\mathbb{C}$, all morphisms are morphisms of schemes, all sheaves will be in the étale topology and cohomology will always refer to étale cohomology. Minor modifications of our methods will prove the analogous results for the uniqueness of holomorphic sections of the adjoint quotient. In general, we will consider sheaves of abelian groups, usually written multiplicatively. The sheaf $\mathbb{G}_{m, T}$ refers to the sheaf (in the étale topology) of sections of the constant group scheme $T \times \mathbb{G}_{m}$. The corresponding sheaf in the Zariski topology will be demoted by $\mathcal{O}_{T}^{*}$. Given a coherent sheaf $\mathcal{F}$ over a scheme $X$, we will also use $\mathcal{F}$ to denote the associated sheaf in the étale topology. We will usually identify a vector bundle with its locally free sheaf of sections. Finally, $E$ always denotes a Weierstrass cubic, necessarily either cuspidal, nodal, or smooth, with a marked smooth point $p_{0}$.

\section{Preliminaries}

\section{$1.1 \quad$ Preliminaries on sheaves}

Definition 1.1.1. Let $X$ be a reduced irreducible scheme and let $\mathcal{S}$ be a sheaf of sets or abelian groups on $\mathcal{S}$.

(i) The sheaf $\mathcal{S}$ is torsion free if, for every étale open set $f: U \rightarrow X$, all sections $s, s^{\prime} \in$ $\Gamma(U, \mathcal{S})$ and every Zariski open dense subset $U^{\prime}$ of $U$, if $s\left|U^{\prime}=s^{\prime}\right| U^{\prime}$, then $s=s^{\prime}$. Equivalently, for every nonempty Zariski open subset $V$ of $X$, if $j: V \rightarrow X$ is the inclusion, then the natural morphism $\mathcal{S} \rightarrow j_{*} j^{*} \mathcal{S}$ is an injection. Since $\mathcal{S}$ is a sheaf in the étale topology, for every étale open set $U \rightarrow X$, if $U^{\prime} \rightarrow U$ is an étale open set with dense image and $s, s^{\prime} \in \Gamma(U, \mathcal{S})$ are such that their restrictions to $U^{\prime}$ are equal, then $s=s^{\prime}$. Note that every subsheaf of a torsion free sheaf is torsion free.

(ii) The sheaf $\mathcal{S}$ has the Hartogs property if for every étale open set $U \rightarrow X$ and every Zariski open subset $U^{\prime} \subseteq U$ whose complement is of codimension at least 2, every section $s \in \Gamma\left(U^{\prime}, \mathcal{S}\right)$ extends to a section $\hat{s} \in \Gamma(U, \mathcal{S})$. If in addition $\mathcal{S}$ is torsion free, then there is a unique such $\hat{s}$. Equivalently, $\mathcal{S}$ has the Hartogs property if for every Zariski open subset $V$ of $X$ such that $X-V$ has codimension at least two, if $j: V \rightarrow X$ is the inclusion, then $\mathcal{S} \rightarrow j_{*} j^{*} \mathcal{S}$ is surjective, and is an isomorphism if in addition $\mathcal{S}$ is torsion free. 
(iii) If $\mathcal{S}$ is a torsion free sheaf of sets over $X$ and $\mathcal{S}^{\prime} \subseteq \mathcal{S}$ is a subsheaf, then $\mathcal{S}^{\prime}$ is a closed subsheaf of $\mathcal{S}$ if for every étale open set $f: U \rightarrow X$, every section $s \in \Gamma(U, \mathcal{S})$ and every étale open dense subset $U^{\prime}$ of $U$, if $s \mid U^{\prime}$ lies in $\Gamma\left(U^{\prime}, \mathcal{S}^{\prime}\right)$, then $s \in \Gamma\left(U, \mathcal{S}^{\prime}\right)$. A standard argument shows that it is enough to check this property for every Zariski open and dense subset $U^{\prime}$ of $U$. If $\mathcal{S}$ is a sheaf of abelian groups and $\mathcal{S}^{\prime}$ is a subsheaf of abelian groups, then $\mathcal{S}^{\prime}$ is a closed subsheaf of $\mathcal{S}$ if and only if the quotient sheaf $\mathcal{S} / \mathcal{S}^{\prime}$ is torsion free.

For example, if $V^{\prime}$ is a subbundle of a vector bundle $V$, then the sheaf of sections of $V^{\prime}$ in the étale topology is a closed subsheaf of the sheaf of sections of $V$.

The following lemma is then clear:

Lemma 1.1.2. (i) Suppose that $\mathcal{S}$ is torsion free and has the Hartogs property. Let $\mathcal{S}^{\prime}$ be a closed subsheaf of $\mathcal{S}$. Then $\mathcal{S}^{\prime}$ is torsion free and has the Hartogs property as well.

(ii) Suppose that $\mathcal{S}^{\prime}$ is a subsheaf of a torsion free sheaf $\mathcal{S}$, and that $\mathcal{C}$ is a closed subsheaf of $\mathcal{S}$. Then $\mathcal{C} \cap \mathcal{S}^{\prime}$ is a closed subsheaf of $\mathcal{S}^{\prime}$.

(iii) If $\mathcal{S}$ and $\mathcal{S}^{\prime}$ are torsion free sheaves of abelian groups and $c: \mathcal{S} \rightarrow \mathcal{S}^{\prime}$ is a homomorphism, then $\operatorname{Ker} c$ is a closed subsheaf of $\mathcal{S}$.

\subsection{A geometric lemma}

Lemma 1.2.1. Let $X$ be a scheme and $T$ a torus such that there exists a morphism $\pi: X \rightarrow$ $T$ and a finite étale covering $\widetilde{T} \rightarrow T$ such that the fiber product $X \times_{T} \widetilde{T}$ is isomorphic to $\mathbb{A}^{k} \times \widetilde{T}$ as schemes over $\widetilde{T}$. Then

(i) Every morphism $f: X \rightarrow \mathbb{G}_{m}$ is induced by a morphism $T \rightarrow \mathbb{G}_{m}$, and hence is given up to translation by an element of $\mathbb{G}_{m}$ by a character of $T$. In particular, every morphism from an affine space to $\mathbb{G}_{m}$ is constant.

(ii) $\operatorname{Pic} X$ is trivial.

Proof. The proof of (i) is left to the reader. To see (ii), let $L$ be a line bundle on $X$ and let $d$ be the degree of the morphism $\widetilde{T} \rightarrow T$. Then the pullback $\widetilde{L}$ of $L$ to $X \times_{T} \widetilde{T}$ is trivial, as is $\operatorname{Norm}(\widetilde{L})=L^{d}$. Thus every element in Pic $X$ is $d$-torsion. Let $(\operatorname{Pic} X)_{d}$ be the group of $d$-torsion line bundles of $X$. The Kummer exact sequence gives an exact sequence

$$
H^{0}\left(X ; \mathbb{G}_{m}\right) \stackrel{z \mapsto z^{d}}{\longrightarrow} H^{0}\left(X ; \mathbb{G}_{m}\right) \rightarrow H^{1}\left(X ; \boldsymbol{\mu}_{d}\right) \rightarrow(\operatorname{Pic} X)_{d} \rightarrow 0 .
$$

Since $X$ is a fiber bundle over $T$ with fibers isomorphic to $\mathbb{A}^{k}$, the comparison of étale and classical cohomology implies that $H^{1}\left(X ; \boldsymbol{\mu}_{d}\right) \cong(\mathbb{Z} / d \mathbb{Z})^{r}$, where $r=\operatorname{dim} T$. By Part (i), $H^{0}\left(X ; \mathbb{G}_{m}\right) \cong \mathbb{C}^{*} \times \mathbf{X}(T)$, where $\mathbf{X}(T)$, the character group of $T$, is a free abelian group 
of rank $r$. Since the homomorphism $H^{0}\left(X ; \mathbb{G}_{m}\right) \rightarrow H^{0}\left(X ; \mathbb{G}_{m}\right)$ is raising to the power $d$, it follows that the cokernel has order $d^{r}$. Hence the cokernel maps isomorphically onto $H^{1}\left(X ; \boldsymbol{\mu}_{d}\right)$ and $(\operatorname{Pic} X)_{d}$ is trivial. Thus Pic $X$ is trivial as well.

\subsection{Minuscule and quasi-minuscule weights}

We begin by recalling some standard definitions from representation theory. The following three sets of objects may be identified: homomorphisms $\Lambda \rightarrow \mathbb{Z}$, linear maps $\mathfrak{h} \rightarrow \mathbb{C}$ which are integral on $\Lambda$, and characters $H \rightarrow \mathbb{C}^{*}$. We will call a homomorphism $\mu: \Lambda \rightarrow \mathbb{Z}$ a weight and will also $\mu$ to denote the obvious extension of $\mu$ to a linear map $\mathfrak{h} \rightarrow \mathbb{C}$ and occasionally the corresponding character $H \rightarrow \mathbb{C}^{*}$.

Definition 1.3.1. Let $\rho: G \rightarrow G L(V)$ be an irreducible representation with highest weight $\varpi$. Then $\rho$ or $\varpi$ is minuscule if for every pair of weights $\mu_{1}, \mu_{2}$ of $\rho$, there exists a $w \in W$ such that $w \mu_{1}=\mu_{2}$. The representation $\rho$ is quasi-minuscule if for every pair of non-zero weights $\mu_{1}, \mu_{2}$ of $\rho$, there exists a $w \in W$ such that $w \mu_{1}=\mu_{2}$. Since highest weights have multiplicity one, if $\rho$ is quasi-minuscule, every non-zero weight of $\rho$ has multiplicity one. Finally, $\rho$ is properly quasi-minuscule if it is quasi-minuscule but not minuscule.

The following is a standard result, cf. [2] :

Lemma 1.3.2. With notation as above,

(i) The minuscule representations are exactly the representations whose highest weight is a fundamental weight $\varpi_{\alpha}$ for a simple root $\alpha$ such that, if $\widetilde{\alpha}=\sum_{\beta \in \Delta} h_{\beta} \beta$ is the highest root, then $h_{\alpha}=1$. Thus, the number of isomorphism classes of minuscule representations is $\# Z(G)-1$, where $Z(G)$ is the center of $G$.

(ii) For each $G$, there is exactly one properly quasi-minuscule representation $\rho$ up to isomorphism. Its highest weight is the highest short root of $G$. Hence, if $G$ is simply laced, $\rho$ is the adjoint representation.

Remark 1.3.3. Let $\rho: G \rightarrow G L(V)$ be a properly quasi-minuscule representation. Let $V=\bigoplus V_{\mu}$ be the decomposition of $V$ into weight spaces, where the $\mu$ are either 0 or the short roots of $G$ and, for $\mu \neq 0, \operatorname{dim} V_{\mu}=1$. Then there exists a non-degenerate $G$-invariant symmetric bilinear form $B: V \otimes V \rightarrow \mathbb{C}$. If $\mu_{1}$ and $\mu_{2}$ are nonzero, then $B\left(V_{\mu_{1}}, V_{\mu_{2}}\right) \neq 0$ if and only if $\mu_{1}+\mu_{2}=0$. There also exists a $G$-invariant 3-tensor $C: V \otimes V \otimes V \rightarrow \mathbb{C}$, with the property that, if $\mu_{1}, \mu_{2}, \mu_{3}$ are nonzero, then $C\left(V_{\mu_{1}}, V_{\mu_{2}}, V_{\mu_{3}}\right) \neq 0$ if and only if $\mu_{1}+\mu_{2}+\mu_{3}=0$. For example, if $G$ is simply laced, then $\rho$ is the adjoint representation. In this case, we let $B(X, Y)$ be the Killing form and let $C(X, Y, Z)$ be the Cartan form $B([X, Y], Z)$. It is easy to see that $B$ and $C$ have the desired properties. The remaining groups are done on a case-by-case basis. For $G=\operatorname{Spin}(2 n+1)$, the representation $\rho$ is the standard representation $G \rightarrow S O(2 n+1) \subseteq G L(2 n+1, \mathbb{C})$. In this case, $B$ is the standard form and $C=0$. (Note that two short roots of $G$ never sum to a short root.) 
If $G=S p(2 n, \mathbb{C})$, let $V_{0}$ be the standard $2 n$-dimensional representation of $G$ with the invariant symplectic form $\langle\cdot, \cdot\rangle$. Then $V$ is a direct summand of $\bigwedge^{2} V_{0}$ complementary to the line defined by $\langle\cdot, \cdot\rangle$. The form $B$ is then the restriction to $V$ of the form on $\bigwedge^{2} V_{0}$ induced by

$$
B\left(v_{1} \wedge v_{2}, w_{1} \wedge w_{2}\right)=\left\langle v_{1}, w_{1}\right\rangle\left\langle v_{2}, w_{2}\right\rangle+\left\langle v_{1}, w_{2}\right\rangle\left\langle v_{2}, w_{1}\right\rangle .
$$

Viewing $C$ as a $G$-invariant homomorphism $\varphi: V \otimes V \rightarrow V^{*} \cong V$ via the form $B$, the tensor $C$ corresponds to

$\varphi\left(v_{1} \wedge v_{2}, w_{1} \wedge w_{2}\right)=\left\langle v_{2}, w_{1}\right\rangle v_{1} \wedge w_{2}-\left\langle v_{1}, w_{1}\right\rangle v_{2} \wedge w_{2}-\left\langle v_{2}, w_{2}\right\rangle v_{1} \wedge w_{1}+\left\langle v_{1}, w_{2}\right\rangle v_{2} \wedge w_{1}$.

The definitions of $B$ and $C$ for the groups of type $G_{2}$ and $F_{4}$ are well-known [1].

In all cases, $\rho(G)$, which is the adjoint form of $G$, is exactly the stabilizer in $G L(V)$ of the forms $B$ and $C$.

While we will not use this remark, it motivates the constructions of Section 5 .

\section{Automorphism sheaves and $G$-bundles}

\subsection{Definition of automorphism sheaves}

Let $X$ and $Y$ be schemes, with $Y$ projective, and let $\Xi \rightarrow X \times Y$ be a principal $G$-bundle over $X \times Y$. We define a sheaf $\pi_{1 *}$ Aut $\Xi$ over $X$ as follows: For each étale open set $f: U \rightarrow X$, let $\Xi_{U}=(f \times \mathrm{Id})^{*} \Xi$ and let $\pi_{1 *}$ Aut $\Xi(U)=$ Aut $\Xi_{U}$. Thus $\pi_{1 *}$ Aut $\Xi(U)$ is the group of sections of $\operatorname{Ad} \Xi_{U}=\Xi_{U} \times_{G} G$, where $G$ acts on itself by conjugation. If $\mathcal{V}$ is a vector bundle over $X \times Y$, the sheaf $\pi_{1 *}$ Aut $\mathcal{V}$ is defined similarly.

There is also the related functor $\mathbf{A}$ from schemes over $X$ to groups defined similarly for each $f: X^{\prime} \rightarrow X$ by $\mathbf{A}\left(X^{\prime}\right)=\operatorname{Aut}(f \times \mathrm{Id})^{*} \Xi$. In this case, one can show the following:

Proposition 2.1.1. Let $E$ be a singular Weierstrass cubic. Suppose that $\Xi \rightarrow X \times E$ is a principal G-bundle such that, for every $x \in X, \Xi \mid\{x\} \times E$ pulls back to the trivial bundle on $\widetilde{E}$ and is regular. Then $\mathbf{A}$ is representable by a commutative affine group scheme which is smooth over $X$.

Since we will not use Proposition 2.1.1 in this paper, we omit the proof.

We now discuss some general properties of $\pi_{1 *}$ Aut $\Xi$ and $\pi_{1 *}$ Aut $\mathcal{V}$. First we have the following:

Lemma 2.1.2. Let $X$ be normal and let $\mathcal{V}$ be a locally free sheaf over $X \times E$. Then $\pi_{1 *} \mathcal{V}$ has the Hartogs property.

Proof. It clearly suffices to show that $\mathcal{O}_{X \times E}$ has the Hartogs property. This is an easy consequence of the fact that $X \times \widetilde{E}$ is normal and that, locally, any section of $\mathcal{O}_{X \times \widetilde{E}}$ which lies in $\mathcal{O}_{X \times E}$ in the complement of a closed subset of codimension at least two lies in $\mathcal{O}_{X \times E}$. 
Lemma 2.1.3. Let $\mathcal{V}$ be a vector bundle over $X \times E$, where $X$ is normal. Then $\pi_{1 *}$ Aut $\mathcal{V}$ is torsion free and has the Hartogs property.

Proof. The sheaf $\pi_{1 *}$ Aut $\mathcal{V}$ is the subsheaf of invertible elements in the sheaf of algebras $\pi_{1 *} \operatorname{Hom}(\mathcal{V}, \mathcal{V})$. Since $\mathcal{V}$ and hence $\operatorname{Hom}(\mathcal{V}, \mathcal{V})$ are vector bundles and $X$ is normal, $\pi_{1 *} \operatorname{Hom}(\mathcal{V}, \mathcal{V})$ is torsion free and has the Hartogs property. Hence $\pi_{1 *}$ Aut $\mathcal{V}$ is torsion free. Suppose that $U \rightarrow X$ is an étale open set and $U^{\prime}=U-Y$ where $Y$ has codimension at least two in $U$. If $s \in \Gamma\left(U^{\prime}, \pi_{1 *}\right.$ Aut $\left.\Xi\right)$, then $s^{-1} \in \Gamma\left(U^{\prime}, \pi_{1 *}\right.$ Aut $\left.\Xi\right)$ as well, and both $s$ and $s^{-1}$ extend to elements of $\Gamma\left(U, \pi_{1 *} \operatorname{Hom}(\mathcal{V}, \mathcal{V})\right)$ whose product is Id on $U^{\prime}$ and hence on $U$. Thus the unique extension of $s$ lies in $\Gamma\left(U, \pi_{1 *}\right.$ Aut $\left.\mathcal{V}\right)$, so that $\pi_{1 *}$ Aut $\mathcal{V}$ has the Hartogs property.

Lemma 2.1.4. Let $\Xi \rightarrow X \times E$ be a principal $G$-bundle over $X \times E$, and suppose that $X$ is normal.

(i) Let $\rho: G \rightarrow \mathbb{C}^{N}$ be a faithful representation and let $\mathcal{V}=\Xi \times_{G} \mathbb{C}^{N}$. Then $\pi_{1 *}$ Aut $\Xi$ is a closed subsheaf of $\pi_{1 *}$ Aut $\mathcal{V}$.

(ii) $\pi_{1 *}$ Aut $\Xi$ is torsion free and has the Hartogs property.

Proof. To see (i), let $f: U \rightarrow X$ be an étale open set and let $\Xi_{U}=(f \times \mathrm{Id})^{*} \Xi$. The scheme $\operatorname{Ad} \Xi_{U}=\Xi_{U} \times{ }_{G} G$ is a closed subscheme of $\Xi_{U} \times_{G} G L(N, \mathbb{C})$. Thus, if $U^{\prime} \rightarrow U$ is a étale open and dense subset of $U$ and $s$ is a section of $\Xi_{U} \times_{G} G L(N, \mathbb{C})$ whose restriction to $U^{\prime} \times E$ lies in $\operatorname{Ad} \Xi_{U^{\prime}}$, then $s$ lies in $\operatorname{Ad} \Xi_{U}$. This proves that $\pi_{1 *}$ Aut $\Xi$ is a closed subsheaf of $\pi_{1 *}$ Aut $\mathcal{V}$. Since $\pi_{1 *}$ Aut $\mathcal{V}$ is torsion free, $\pi_{1 *}$ Aut $\Xi$ is torsion free as well, and by Lemma 1.1 .2 and Lemma 2.1.3, $\pi_{1 *}$ Aut $\Xi$ has the Hartogs property.

\subsection{Statement of the main theorem}

We now specialize to the case of a singular Weierstrass cubic $E$. Recall the notation of the introduction: let $\widetilde{\mathcal{M}}=E_{\text {reg }} \otimes \Lambda$, so that $\widetilde{\mathcal{M}}=\mathfrak{h}$ if $E$ is cuspidal and $\widetilde{\mathcal{M}}=H$ if $E$ is nodal, and let $\mathcal{M}=\widetilde{\mathcal{M}} / W$ be the adjoint quotient.

Definition 2.2.1. Fix once and for all a section of the adjoint quotient morphism, for example the Kostant or Steinberg section, and let $\Xi \rightarrow \mathcal{M} \times E$ be the corresponding $G$-bundle. Let $\mathcal{A}=\pi_{1 *}$ Aut $\Xi$, where $\Xi \rightarrow \mathcal{M} \times E$ is a universal $G$-bundle as above.

Since the centralizer of a regular element is abelian, $\mathcal{A}$ is a sheaf of abelian groups over $\mathcal{M}$. It is easy to see that two different choices of a section lead to isomorphic automorphism sheaves. Note further that there is a natural inclusion of the center $Z(G)$ of $G$, viewed as a constant sheaf on $\mathcal{M}$, into $\mathcal{A}$.

If $\sigma^{\prime}$ is another section of the adjoint quotient morphism, let $\Xi^{\prime}$ be the corresponding bundle. Then, for all $x \in \mathcal{M}$, there exists a $g \in G$ such that $\operatorname{ad}(g) \sigma(x)=\sigma^{\prime}(x)$, and any two 
such differ by right multiplication by $Z_{G}(\sigma(x))$. Since $\sigma$ is a section of the adjoint quotient morphism, the morphism $G \times \mathfrak{h} / W \rightarrow \mathfrak{g}_{\text {reg }}$ defined by $(g, x) \mapsto \operatorname{ad} g(\sigma(x))$ is smooth. Thus there exist local sections of this morphism in the étale topology. Similarly in the case of the adjoint quotient of $G$, the morphism $G \times H / W \rightarrow G_{\text {reg }}$ defined by $(g, x) \mapsto \operatorname{Ad} g(\sigma(x))$ is smooth. A formal argument then shows:

Proposition 2.2.2. Let $\sigma$ and $\sigma^{\prime}$ be two sections of the adjoint quotient morphism $\mathfrak{g}_{\mathrm{reg}} \rightarrow$ $\mathfrak{h} / W=\mathcal{M}$ or $G_{\text {reg }} \rightarrow H / W=\mathcal{M}$. For every $x \in \mathcal{M}$, there exists an étale neighborhood $U$ of $x$ and a function $\gamma: U \rightarrow G$ such that $\sigma^{\prime} \mid U=\operatorname{ad}(\gamma)(\sigma \mid U)$, in the case of $\mathfrak{g}$, or $\sigma^{\prime} \mid U=\operatorname{Ad}(\gamma)(\sigma \mid U)$ in the case of $G$. Moreover, any two choices of $\gamma$ differ by right multiplication by an element of $\mathcal{A}(U)$.

In the standard way, choosing a sufficiently small open cover $\left\{U_{i}\right\}$ of $\mathcal{M}$ and writing $\sigma^{\prime} \mid U_{i}=\operatorname{ad}\left(\gamma_{i}\right)\left(\sigma \mid U_{i}\right)$ with $\gamma_{j}\left|U_{i} \times{ }_{\mathcal{M}} U_{j}=\gamma_{i} a_{i j}\right| U_{i} \times_{\mathcal{M}} U_{j}$ defines a 1-cocycle $\left\{a_{i j}\right\}$ and hence an element of $H^{1}(\mathcal{M} ; \mathcal{A})$ which is independent of the choices. This element is the identity if and only if the bundles $\Xi$ and $\Xi^{\prime}$ are isomorphic, if and only if there exists a morphism $g: \mathcal{M} \rightarrow G$ such that $\sigma^{\prime}=\operatorname{ad} g(\sigma)$, in which case we say that the sections $\sigma$ and $\sigma^{\prime}$ are conjugate. This explains the relevance of the second half of the following theorem:

Theorem 2.2.3. Let $\mathcal{M}$ be the adjoint quotient of either $\mathfrak{g}$ or $G$, and let $\mathcal{A}$ denote the automorphism sheaf corresponding to the section $\sigma$ with image contained in the set of regular elements. Then

$$
H^{0}(\mathcal{M} ; \mathcal{A})= \begin{cases}Z(G), & \text { for the case of } \mathfrak{g} \\ Z(G) \times \mathbb{Z}, & \text { for the case of } G\end{cases}
$$

Moreover, in both cases, $H^{1}(\mathcal{M} ; \mathcal{A})=\{1\}$.

Corollary 2.2.4. Every two sections of the adjoint quotient morphism are conjugate by a morphism from the adjoint quotient to the set of regular elements of $\mathfrak{g}$ or $G$.

\section{Quasi-minuscule representations and spectral covers}

\subsection{Vector bundles attached to quasi-minuscule representations}

We fix the following notation: $\rho: G \rightarrow \operatorname{Aut}(V)$ is a quasi-minuscule representation, with highest weight $\varpi$ (with respect to the given fixed set of simple roots). Let $W_{0} \subseteq W$ be the stabilizer of $\varpi$. The group $W_{0}$ is the group generated by reflections in the roots annihilated by $\varpi$.

With $\mathcal{M}$ as before, and $\Xi$ the universal bundle over $\mathcal{M} \times E$ corresponding to the choice of the section $\sigma$, let $\mathcal{V} \rightarrow \mathcal{M} \times E$ be the vector bundle induced by the representation $\rho$. By [10, Theorem 2.4.3], we have:

Proposition 3.1.1. The sheaf $\pi_{1 *} \mathcal{V}$ is a locally free sheaf over $\mathcal{M}$ whose rank is the multiplicity of the trivial weight in $\rho$. Moreover, the inclusion $\pi_{1}{ }^{*} \pi_{1 *} \mathcal{V} \rightarrow \mathcal{V}$ is a subbundle, 
so that the quotient $\overline{\mathcal{V}}$ is a vector bundle, and there is an exact sequence of vector bundles over $\mathcal{M} \times E$ :

$$
0 \rightarrow \pi_{1}^{*} \pi_{1 *} \mathcal{V} \rightarrow \mathcal{V} \rightarrow \overline{\mathcal{V}} \rightarrow 0 \text {. }
$$

We shall need the following facts about $\Xi$ and $\mathcal{V}$ :

Lemma 3.1.2. There is an étale open and dense subset $U \rightarrow \mathcal{M}$ such that $\Xi_{U}$ has a reduction of structure to an $H$-bundle $\Xi_{U, H}$. The action of the constant group scheme $U \times H$ over $U$ as a group scheme of automorphisms of $\Xi_{U, H}$ defines an isomorphism from the sheaf of sections of the constant group scheme $U \times H \rightarrow U$ to $\mathcal{A} \mid U$.

Proof. Let $U$ be the nonempty open subset of $\widetilde{\mathcal{M}}$ where no root is the identity. Thus $U$ is either $\mathfrak{h}_{\text {reg }}=\mathfrak{h} \cap \mathfrak{g}_{\text {reg }}$ or $H_{\text {reg }}=H \cap G_{\text {reg. }}$. We just write out the Lie algebra case. The induced morphism $U \rightarrow \mathcal{M}$ is étale. For a scheme $B$, an $H$-bundle over $B \times E$ which is trivialized over $B \times \widetilde{E}$ is identified with a morphism $B \rightarrow \mathfrak{h}$. Let $\Xi_{H, U}$ be the $H$-bundle over $U \times E$ induced by applying this remark to the inclusion $U=\mathfrak{h}_{\text {reg }} \rightarrow \mathfrak{h}$ and let $\Xi_{U}^{\prime}=\Xi_{H, U} \times_{H} G$. It is clear that $\Xi_{U}$ and $\Xi_{U}^{\prime}$ have isomorphic restriction to every slice. A formal argument as in the proof of Proposition 2.2.2 shows that, possibly after replacing $U$ by an étale open dense subset, the bundles $\Xi_{U}$ and $\Xi_{U}^{\prime}$ become isomorphic. This gives the desired reduction of structure. Viewing $\Xi_{U} \cong \Xi_{U}^{\prime}$ as the $G$-bundle associated to a morphism $\sigma^{\prime}: U \rightarrow \mathfrak{h}_{\text {reg }} \subseteq \mathfrak{g}$, the sections of $\mathcal{A}$ over an étale open set $V$ of $U$ are given by morphisms $f: V \rightarrow G$ such that $\operatorname{ad}(f)\left(\sigma^{\prime} \mid V\right)=\sigma^{\prime} \mid V$. Clearly, since the image of $\sigma^{\prime}$ is contained in $\mathfrak{h}_{\text {reg }}$, this is the same as the group of morphisms from $V$ to $H$.

Corollary 3.1.3. Let $U \rightarrow \mathcal{M}$ be the étale open subset of Lemma 3.1.9. Then $\mathcal{V} \mid U \times E$ is isomorphic to $\bigoplus_{\mu} \mathcal{L}_{\mu}^{\oplus m_{\mu}}$, where the $\mu$ are the weights of $\rho, m_{\mu}$ is the multiplicity of $\mu$ in $\rho$, and the $\mathcal{L}_{\mu}$ are line bundles on $U \times E$ with the property that $\mathcal{L}_{\mu} \cong \mathcal{L}_{\mu^{\prime}}$ if and only if $\mu=\mu^{\prime}$. Under the isomorphism of $\mathcal{A} \mid U$ with the sheaf of sections of the constant group scheme $U \times H$, a local section s of $U \times H$ acts on $\mathcal{L}_{\mu}^{\oplus m_{\mu}}$ via multiplication by the function $\mu(s)$.

As before, let $\mathcal{A}$ be the automorphism sheaf of $\Xi$. The representation $\rho$ induces a homomorphism $\mathcal{A} \rightarrow \pi_{1 *} \operatorname{Aut}(\mathcal{V})$. The kernel of this homomorphism is the subsheaf of constant automorphisms with values in $\operatorname{Ker}(\rho) \subseteq Z(G)$. Let $\overline{\mathcal{A}}_{\rho}$ denote the quotient sheaf $\mathcal{A} / \operatorname{Ker}(\rho)$.

Lemma 3.1.4. In the above notation, there is an exact sequence

$$
\{1\} \rightarrow \operatorname{Ker}(\rho) \rightarrow H^{0}(\mathcal{M} ; \mathcal{A}) \rightarrow H^{0}\left(\mathcal{M} ; \overline{\mathcal{A}}_{\rho}\right) \rightarrow\{1\}
$$

and the natural map $H^{1}(\mathcal{M} ; \mathcal{A}) \rightarrow H^{1}\left(\mathcal{M} ; \overline{\mathcal{A}}_{\rho}\right)$ is an isomorphism.

Proof. This is an immediate consequence of the fact that, as $\mathcal{M}$ is isomorphic to $\mathbb{A}^{r}$, $H^{0}(\mathcal{M} ; \operatorname{Ker}(\rho)) \cong \operatorname{Ker}(\rho)$ and $H^{i}(\mathcal{M} ; \operatorname{Ker}(\rho))$ is trivial for $i=1,2$. 
Proposition 3.1.5. The sheaves $\mathcal{A}$ and $\overline{\mathcal{A}}_{\rho}$ are torsion free and have the Hartogs property. The sheaf $\overline{\mathcal{A}}_{\rho}$ is a closed subsheaf of $\pi_{1 *} \operatorname{Aut}(\mathcal{V})$. Finally, every local section of $\overline{\mathcal{A}}_{\rho}$ induces the identity on $R^{0} \pi_{1 *} \mathcal{V}$.

Proof. The first two statements follow from Lemma 2.1.4. It suffices to prove the final statement on an étale open dense of $\mathcal{M}$. By Corollary 3.1.3, it is immediate that $\overline{\mathcal{A}}_{\rho}$ has this property on the étale open set $U \rightarrow \mathcal{M}$ of Lemma 3.1.2.

\subsection{The spectral cover}

We begin by recalling the general formalism of the Fourier-Mukai correspondence in the spcecial case we shall need, for which a general reference is [10, §1]. Let $B$ be a scheme and let $\mathcal{V}$ be a vector bundle of rank $n$ over $B \times E$ whose restriction to every slice $\{x\} \times E$ has trivial pullback to the normalization of $E$. Then $\mathcal{V}$ determines functorially a relative Cartier divisor $T_{\mathcal{V}} \subseteq B \times E_{\text {reg }}$, finite over $B$, and a sheaf $\mathcal{Q}_{\mathcal{V}}$ of $\mathcal{O}_{T_{\mathcal{V}}}$-modules, finite and flat over $B$ of degree $n$, such that $\mathcal{V}=q_{*}\left(p_{1}^{*}\left(\mathcal{Q}_{\mathcal{V}} \otimes \pi_{2}^{*} \mathcal{O}_{E}\left(p_{0}\right)\right) \otimes r^{*} \mathcal{P}\right)$, where $p_{1}: B \times E \times E$ is projection onto the first two factors, $q: B \times E \times E$ is projection onto the first and third factors, $r: B \times E \times E \rightarrow E \times E$ is projection onto the last two factors, and $\pi_{2}: B \times E \rightarrow E$ is projection onto the second factor. The divisor $T_{\mathcal{V}}$ is additive under exact sequences, and, viewing $\mathcal{Q V}_{\mathcal{V}}$ as a sheaf on $B \times E$, given an exact sequence $0 \rightarrow \mathcal{V}^{\prime} \rightarrow \mathcal{V} \rightarrow \mathcal{V}^{\prime \prime} \rightarrow 0$ of vector bundles as above, there is an exact sequence of the corresponding sheaves:

$$
0 \rightarrow \mathcal{Q}_{\mathcal{V}^{\prime}} \rightarrow \mathcal{Q}_{\mathcal{V}} \rightarrow \mathcal{Q}_{\mathcal{V}^{\prime \prime}} \rightarrow 0
$$

Moreover the constructions of $T_{\mathcal{V}}$ and $\mathcal{Q}_{\mathcal{V}}$ are compatible with base change. We somewhat loosely refer to $T_{\mathcal{V}}$ as the spectral cover of $B$ corresponding to $\mathcal{V}$. More generally, we have the following:

Definition 3.2.1. Let $\nu: T \rightarrow B$ be a finite morphism of schemes. Suppose that $\mathcal{Q}$ is a coherent sheaf on $T$, flat over $B$, and that $\mathcal{L}$ is a line bundle on $T \times E$. The spectral cover construction (applied to $\mathcal{Q}$ and $\mathcal{L}$ ) is the vector bundle

$$
\mathcal{V}=(\nu \times \operatorname{Id})_{*}\left(\pi_{1}^{*} \mathcal{Q} \otimes \mathcal{L}\right)
$$

In the above situation, the sheaf $\nu_{*} \mathcal{O}_{T}$ acts via endomorphisms on $\mathcal{V}$, and hence $\nu_{*} \mathcal{O}_{T}^{*}$ acts via automorphisms of $\mathcal{V}$. Passing from a sheaf in the Zariski topology to one in the étale topology defines a homomorphism $\nu_{*} \mathbb{G}_{m, T} \rightarrow \pi_{1 *}$ Aut $\mathcal{V}$.

Lemma 3.2.2. In the above notation, suppose that $B$ is reduced irreducible and that $\mathcal{Q}$ is a locally free $\mathcal{O}_{T}$-module, or more generally that $T$ is flat over $B$ and that, for all $x \in B$, $H^{0}\left(\nu^{-1}(x) ; \mathcal{Q} \mid \nu^{-1}(x)\right)$ is a faithful $H^{0}\left(\nu^{-1}(x) ; \mathcal{O}_{T} \mid \nu^{-1}(x)\right)$-module. Then $\nu_{*} \mathbb{G}_{m, T}$ is a closed subsheaf of $\pi_{1 *}$ Aut $\mathcal{V}$. 
Proof. The fiber of $\mathcal{V}$ over a point $(x, e)$ of $B \times E$ is identified up to a homothety with $H^{0}\left(\nu^{-1}(x) ; \mathcal{Q} \mid \nu^{-1}(x)\right)$ and the induced action of $\nu_{*} \mathcal{O}_{T}$ on this fiber is via the action of $H^{0}\left(\nu^{-1}(x) ; \mathcal{O}_{T} \mid \nu^{-1}(x)\right)$. Since $T$ is flat over $B, \nu_{*} \mathcal{O}_{T}$ is a vector bundle. The hypotheses imply that, for each $x \in B,\left(\nu_{*} \mathcal{O}_{T}\right)_{x} \rightarrow \pi_{1 *} \operatorname{Hom}(\mathcal{V}, \mathcal{V})_{x}$ is injective. Thus $\nu_{*} \mathcal{O}_{T}$ is a vector subbundle, and hence a closed subsheaf, of $\pi_{1 *} \operatorname{Hom}(\mathcal{V}, \mathcal{V})$. By (i) of Lemma 1.1.2, as sheaves of sets in the étale topology, $\pi_{1 *}$ Aut $\mathcal{V} \cap \nu_{*} \mathcal{O}_{T}$ is a closed subsheaf of $\pi_{1 *}$ Aut $\mathcal{V}$. It suffices to prove that $\pi_{1 *}$ Aut $\mathcal{V} \cap \nu_{*} \mathcal{O}_{T}=\nu_{*} \mathbb{G}_{m, T}$. Replacing $B$ by an étale open set and $T$ by the fiber product, it is enough to show that, if $f \in \Gamma\left(T, \mathcal{O}_{T}\right)$ defines an automorphism of $\mathcal{V}$ and hence by functoriality of $\mathcal{Q}$, then, for all $y \in T, f$ does not lie in the maximal ideal $\mathfrak{m}_{y}$ of $y$. Since $f$ defines an automorphism of $\mathcal{Q}$, it defines an automorphism of the stalk of $\mathcal{Q}$ over $y$, which is a finite module over the local ring of $T$ at $y$ and is nonzero by our assumptions on $\mathcal{Q}$. It follows by Nakayama's lemma that $f \notin \mathfrak{m}_{y}$. Thus $\pi_{1 *}$ Aut $\mathcal{V} \cap \nu_{*} \mathcal{O}_{T}=\nu_{*} \mathbb{G}_{m, T}$ is a closed subsheaf of $\pi_{1 *}$ Aut $\mathcal{V}$.

\subsection{The case of a quasi-minuscule representation}

We return to the case where $\mathcal{V}=\Xi \times{ }_{G} \mathbb{C}^{N}$ is the vector bundle over $\mathcal{M} \times E$ defined by the minuscule or quasi-minuscule representation $\rho: G \rightarrow G L(N, \mathbb{C})$. Recall that we have the exact sequence

$$
0 \rightarrow \pi_{1}{ }^{*} \pi_{1 *} \mathcal{V} \rightarrow \mathcal{V} \rightarrow \overline{\mathcal{V}} \rightarrow 0
$$

Let $\hat{T}_{0}=\widetilde{\mathcal{M}} / W_{0}$ and let $\hat{\nu}: \hat{T}_{0} \rightarrow \mathcal{M}$ be the natural morphism. The homomorphism $\varpi: \Lambda \rightarrow \mathbb{Z}$ defines a $W_{0}$-invariant homomorphism $\widetilde{\mathcal{M}} \rightarrow E_{\text {reg }}$ and hence a morphism $f_{\varpi}: \hat{T}_{0} \rightarrow E_{\text {reg. }}$. Let $T_{0} \subseteq \mathcal{M} \times E_{\text {reg }}$ be the image of the product morphism $\left(\hat{\nu}, f_{\varpi}\right)$. By [10, Corollary 2.2.3], the morphism $\hat{T}_{0} \rightarrow T_{0}$ is birational and exhibits $\hat{T}_{0}$ as the normalization of $T_{0}$. Moreover, by [10, Lemma 2.1.1], we have:

Lemma 3.3.1. Let $\Lambda_{0}=\operatorname{Ker} \varpi$ and let $\mathcal{M}_{0}=\left(E_{\mathrm{reg}} \otimes \Lambda_{0}\right) / W_{0}$. There is a finite étale cover $C \rightarrow E_{\mathrm{reg}}$, with $C$ necessarily isomorphic to $E_{\mathrm{reg}}$, such that $\hat{T}_{0} \times_{E_{\mathrm{reg}}} C \cong \mathcal{M}_{0} \times C$ as schemes over $C$.

Let $T=T_{\mathcal{V}} \subseteq \mathcal{M} \times E_{\text {reg }}$ be the spectral cover of $\mathcal{V}$, let $\nu: T \rightarrow \mathcal{M}$ be the morphism induced by projection onto the first factor, and let $\mathcal{Q}=\mathcal{Q} \mathcal{V}$ be the corresponding sheaf of $\mathcal{O}_{T}$-modules. Let $\mathcal{Q}_{1}$ be the sheaf corresponding to $\pi_{1}{ }^{*} \pi_{1 *} \mathcal{V}$ and let $\overline{\mathcal{Q}}$ be the sheaf corresponding to $\overline{\mathcal{V}}$. The general formalism of the previous subsection and [10, Corollary 2.2.3] imply:

Proposition 3.3.2. Let $T_{0}$ be the divisor defined above, let $\bar{T}_{1}$ be the reduced divisor $\mathcal{M} \times$ $\left\{p_{0}\right\}$, and let $m_{0}$ be the multiplicity of the trivial weight in $\rho$. Then the divisor $T_{0}$ is the spectral cover of $\overline{\mathcal{V}}$, and $T=T_{0} \cup T_{1} \subset \mathcal{M} \times E_{\text {reg }}$ where, as a divisor, $T_{1}=m_{0} \cdot \bar{T}_{1}$. Thus $T=T_{0}$ if and only if $\rho$ is minuscule. Generically along $\bar{T}_{1} \cap T_{0}$ the varieties $\bar{T}_{1}$ and $T_{0}$ are smooth and meet transversely. 
Let $\bar{T}$ be the sum $T_{0}+\bar{T}_{1}$ considered as a (reduced) effective Cartier divisor of $\mathcal{M} \times E$. It is the reduced subscheme of $T$. By the above, the following is exact on the complement of a codimension two subset of $\bar{T}$ :

$$
0 \rightarrow \mathcal{O}_{\bar{T}} \rightarrow \mathcal{O}_{\bar{T}_{1}} \oplus \mathcal{O}_{T_{0}} \rightarrow \mathcal{O}_{\bar{T}_{1} \cap T_{0}} \rightarrow 0
$$

In fact, since $\bar{T}$ is Cohen-Macaulay, the above sequence is everywhere exact, although we will not need this.

Proposition 3.3.3. The sheaf $\mathcal{Q}$ of $\mathcal{O}_{T}$-modules is in fact a sheaf of $\mathcal{O}_{\bar{T}}$-modules.

Proof. By the formalism of spectral covers, there is an exact sequence

$$
0 \rightarrow \mathcal{Q}_{1} \rightarrow \mathcal{Q} \rightarrow \overline{\mathcal{Q}} \rightarrow 0
$$

Since $\pi_{1}{ }^{*} \pi_{1 *} \mathcal{V}$ is pulled back from a bundle on $\mathcal{M}$, it restricts to the trivial bundle on every slice and hence $\mathcal{Q}_{1}$ is annihilated by $I_{\bar{T}_{1}}$, the ideal sheaf of $\bar{T}_{1}$. Since $\overline{\mathcal{Q}}$ is an $\mathcal{O}_{T_{0}}$-module, it is annihilated by the ideal sheaf $I_{T_{0}}$. Hence $\mathcal{Q}$ is annihilated by the product ideal $I_{\bar{T}_{1}} \cdot I_{T_{0}}$, which is the ideal of $\bar{T}$.

As for the quotient itself, by [10, Theorem 2.4.3] and Lemma 1.2.1, we have:

Proposition 3.3.4. The sheaf $\overline{\mathcal{Q}}$ is isomorphic to an invertible $\mathcal{O}_{\hat{T}_{0}}$-module, and hence is isomorphic, as a sheaf of $\mathcal{O}_{T_{0}}$-modules, to $\mathcal{O}_{\hat{T}_{0}}$.

Corollary 3.3.5. $\pi_{1 *}$ Aut $\overline{\mathcal{V}}=\hat{\nu}_{*} \mathbb{G}_{m, \hat{T}_{0}}$. In particular, if $\rho$ is minuscule, then $\mathcal{V}=\overline{\mathcal{V}}$, $\pi_{1 *}$ Aut $\mathcal{V}=\hat{\nu}_{*} \mathbb{G}_{m, \hat{T}_{0}}$, and $\overline{\mathcal{A}}_{\rho}$ is a closed subsheaf of $\hat{\nu}_{*} \mathbb{G}_{m, \hat{T}_{0}}$.

Proof. By Lemma 3.2.2, $\hat{\nu}_{*} \mathbb{G}_{m, \hat{T}_{0}}$ is a closed subsheaf of $\pi_{1 *}$ Aut $\overline{\mathcal{V}}$. Thus, it suffices to prove that they agree on an étale open and dense set. By Corollary 3.1.3, there is an étale open set $U$ such that the pullback of $\mathcal{V}$ to $U$ is of the form $\bigoplus_{\mu} \mathcal{L}_{\mu}^{\oplus m_{\mu}}$, where the $\mathcal{L}_{\mu}$ are distinct line bundles of relative degree zero on $U \times E$, indexed by the weights of $\rho$, and the $m_{\mu}$ are the multiplicities and thus $m_{\mu}=1$ if $\mu \neq 0$. Hence $\overline{\mathcal{V}} \mid U \times E \cong \bigoplus_{\mu \neq 0} \mathcal{L}_{\mu}$. Thus $\pi_{1 *}$ Aut $\overline{\mathcal{V}} \mid U$ is just a product of copies of $\mathbb{G}_{m, U}$ indexed by the nonzero weights of $\rho$, and this is clearly equal to $\hat{\nu}_{*} \mathbb{G}_{m, \hat{T}_{0}} \mid U$.

\subsection{The properly quasi-minuscule case}

In the properly quasi-minuscule case, we will need a refinement of Corollary 3.3.5. Thus throughout this subsection we assume that $\rho$ is properly quasi-minuscule. We have identified $\hat{T}_{0}$ with $\widetilde{\mathcal{M}} / W_{0}$. Let $D=\bar{T}_{1} \cap T_{0}$ and let $\hat{D}$ be the preimage of $D$ in $\hat{T}_{0}$. The preimage of $\hat{D}$ in $\widetilde{\mathcal{M}}$ is the kernel of the weight $\varpi$, and $\hat{D}=f_{\varpi}^{-1}\left(p_{0}\right)$ as reduced divisors. If $\Lambda_{0} \subseteq \Lambda$ is the kernel of $\varpi$, then $W_{0}$ acts on $E_{\text {reg }} \otimes \Lambda_{0}$ and $\hat{D}=\left(E_{\text {reg }} \otimes \Lambda_{0}\right) / W_{0} \subseteq \hat{T}_{0}$. 
Lemma 3.4.1. Let $X=\pi_{1}(D) \cap \pi_{1}\left(\left(T_{0}\right)_{\text {sing }}\right)$. Then $X$ has codimension at least two in $\mathcal{M}$. Moreover, the morphism $\hat{D} \rightarrow D$ is birational and identifies $\hat{D}$ with the normalization of $D$.

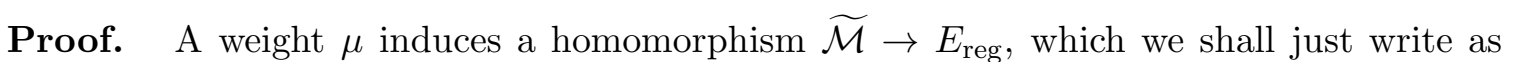
$\mu$ again. By [10, Lemma 2.3.5], there is an open dense subset of the preimage of $\left(T_{0}\right)_{\text {sing }}$ in $\widetilde{\mathcal{M}}$ consisting of points $x$ such that $\mu_{1}(x)=\mu_{2}(x)$ for two distinct weights $\mu_{1}, \mu_{2}$ of $\rho$ and such that no root $\alpha$ is the identity on $x$. The inverse image of $D$ in $\widetilde{\mathcal{M}}$ is the set of $x$ such that some nonzero weight of $\rho$ is the identity on $x$. Since the nonzero weights of $\rho$ are roots, the inverse image of $\pi_{1}(D) \cap \pi_{1}\left(\left(T_{0}\right)_{\text {sing }}\right)$ in $\widetilde{\mathcal{M}}$ has codimension at least two in $\widetilde{\mathcal{M}}$, and since $\widetilde{\mathcal{M}} \rightarrow \mathcal{M}$ is finite, the same is true for $X$. The final statement is then clear.

Let $\hat{T}$ be the scheme $\hat{T}_{0} \amalg \bar{T}_{1} / \sim$, where $\sim$ is the identification of a point in $\hat{D}$ with its image in $D$. Thus the normalization of $\hat{T}$ is $\hat{T}_{0} \amalg \bar{T}_{1}$, and the morphism $\hat{T}_{0} \rightarrow T_{0}$ induces a morphism $\hat{T} \rightarrow \bar{T}$, and hence an algebra homomorphism $\mathcal{O}_{\bar{T}} \rightarrow \mathcal{O}_{\hat{T}}$.

Proposition 3.4.2. The sheaf $\mathcal{Q}$ is a sheaf of $\mathcal{O}_{\hat{T}}$-modules, compatibly with its structure as a sheaf of $\mathcal{O}_{\bar{T}}$-modules.

Proof. The sheaf $\pi_{1 *} H o m(\mathcal{V}, \mathcal{V})$ is torsion free and has the Hartogs property. Thus, if there is an algebra homomorphism $\hat{\nu}_{*} \mathcal{O}_{\hat{T}} \rightarrow \pi_{1 *} \operatorname{Hom}(\mathcal{V}, \mathcal{V})$ on the complement $U$ of a closed subset of codimension at least two, then it extends to a homomorphism over $\hat{T}$, by taking the composition

$$
\hat{\nu}_{*} \mathcal{O}_{\hat{T}} \rightarrow j_{*} j^{*} \hat{\nu}_{*} \mathcal{O}_{\hat{T}} \rightarrow j_{*} j^{*} \pi_{1 *} \operatorname{Hom}(\mathcal{V}, \mathcal{V}) \cong \pi_{1 *} \operatorname{Hom}(\mathcal{V}, \mathcal{V})
$$

where $j: U \rightarrow \mathcal{M}$ is the inclusion.

Let $X=\pi_{1}(D) \cap \pi_{1}\left(\left(T_{0}\right)_{\text {sing }}\right)$ and let $U=\mathcal{M}-X$. By Lemma 3.4.1, $X$ is a codimension two subset of $\mathcal{M}$. Set $U_{1}=\mathcal{M}-\pi_{1}\left(\left(T_{0}\right)_{\text {sing }}\right)$ and $U_{2}=\mathcal{M}-\pi_{1}(D)$. Then $U=U_{1} \cup U_{2}$, and it will suffice to check that $\mathcal{Q}$ is a sheaf of $\mathcal{O}_{\hat{T}}$-modules over both $U_{1}$ and $U_{2}$. Over $U_{1}$, $\hat{T}=\bar{T}$ and we conclude by Proposition 3.3.3. Over $U_{2}, \bar{T}$ is a disjoint union of $\bar{T}_{1}$ and $T_{0}$. Hence $\mathcal{Q} \mid U_{2}$ is a direct sum $\mathcal{Q}_{1}\left|U_{2} \oplus \overline{\mathcal{Q}}\right| U_{2}$, and we conclude by Proposition 3.3.4.

We define the sheaf $\mathcal{B}_{\hat{D}}$ to be the subsheaf of $\mathbb{G}_{m, \hat{T}_{0}}$ such that

$$
\mathcal{B}_{\hat{D}}(U)=\left\{f \in \Gamma\left(U, \mathcal{O}_{U}^{*}\right): f \mid U \times_{\hat{T}_{0}} \hat{D}=1\right\} .
$$

Thus $\mathcal{B}_{\hat{D}}$ is the kernel of the restriction homomorphism $\mathbb{G}_{m, \hat{T}_{0}} \rightarrow i_{*} \mathbb{G}_{m, \hat{D}}$, where $i$ is the inclusion of $\hat{D}$ in $\hat{T}_{0}$ and there is a defining exact sequence

$$
\{1\} \rightarrow \mathcal{B}_{\hat{D}} \rightarrow \mathbb{G}_{m, \hat{T}_{0}} \rightarrow i_{*} \mathbb{G}_{m, \hat{D}} \rightarrow\{1\} .
$$

Clearly, $\hat{\nu}_{*} \mathcal{B}_{\hat{D}}$ has the Hartogs property. 
Lemma 3.4.3. The sheaf $\hat{\nu}_{*} \mathcal{B}_{\hat{D}}$ is naturally a subsheaf of $\pi_{1 *} \operatorname{Aut}(\mathcal{V})$.

Proof. Since $T_{0}$ and $\bar{T}_{1}$ meet generically transversely in $D$, an invertible function on $\hat{T}_{0}$ which is 1 along $\hat{D}$ extends to a function on $\hat{T}$ by defining it to be 1 on $\bar{T}_{1}$. Thus inclusion induces an isomorphism from $\hat{\nu}_{*} \mathcal{B}_{\hat{D}}$ to the sheaf of functions on $\hat{T}$ that are identically 1 on $\bar{T}_{1}$, and hence there is an inclusion $\hat{\nu}_{*} \mathcal{B}_{\hat{D}} \rightarrow \nu_{*} \mathbb{G}_{m, \hat{T}}$. Since by Proposition 3.4 .2 the sheaf $\mathcal{Q}$ is a sheaf of $\mathcal{O}_{\hat{T}}$-modules, there is a homomorphism $\nu_{*} \mathbb{G}_{m, \hat{T}} \rightarrow \pi_{1 *} \operatorname{Aut}(\mathcal{V})$ and hence a homomorphism $\hat{\nu}_{*} \mathcal{B}_{\hat{D}} \rightarrow \pi_{1 *} \operatorname{Aut}(\mathcal{V})$. It is easy to check that this homomorphism is injective.

Lemma 3.4.4. Let $\mathrm{Aut}^{\prime} \mathcal{V}$ be the sheaf of automorphisms of $\mathcal{V}$ which are the identity on the subbundle $\pi_{1}{ }^{*} \pi_{1 *} \mathcal{V}$, so that there is a natural homomorphism Aut $^{\prime} \mathcal{V} \rightarrow$ Aut $\overline{\mathcal{V}}$. Then there is an isomorphism $\hat{\nu}_{*} \mathcal{B}_{\hat{D}} \rightarrow \pi_{1 *}$ Aut $^{\prime} \mathcal{V}$ such that the composition

$$
\hat{\nu}_{*} \mathcal{B}_{\hat{D}} \rightarrow \pi_{1 *} \operatorname{Aut}^{\prime} \mathcal{V} \rightarrow \pi_{1 *} \text { Aut } \overline{\mathcal{V}}=\hat{\nu}_{*} \mathbb{G}_{m, \hat{T}_{0}}
$$

is the inclusion.

Proof. The previous lemma established an inclusion of $\hat{\nu}_{*} \mathcal{B}_{\hat{D}}$ in $\pi_{1 *} \operatorname{Aut}(\mathcal{V})$. Since the image of $\hat{\nu}_{*} \mathcal{B}_{\hat{D}}$ is contained in the sheaf of functions on $\hat{T}$ that are identically 1 on $\bar{T}_{1}$, the image of $\hat{\nu}_{*} \mathcal{B}_{\hat{D}}$ in $\pi_{1 *} \operatorname{Aut}(\mathcal{V})$ is contained in $\pi_{1 *} \operatorname{Aut}^{\prime} \mathcal{V}$. To find a homomorphism in the other direction, we use:

Claim 3.4.5. The image of $\pi_{1 *} \operatorname{Aut}^{\prime} \mathcal{V}$ in $\pi_{1 *}$ Aut $\overline{\mathcal{V}} \cong \hat{\nu}_{*} \mathbb{G}_{m, \hat{T}_{0}}$ is contained in $\hat{\nu}_{*} \mathcal{B}_{\hat{D}}$

Proof. Since $\hat{\nu}_{*} \mathcal{B}_{\hat{D}}$ has the Hartogs property and $\hat{\nu}_{*} \mathbb{G}_{m, \hat{T}_{0}}$ is torsion free, it suffices to establish the containment on the complement of a codimension two subset of $\mathcal{M}$. As before, let $X=\pi_{1}(D) \cap \pi_{1}\left(\left(T_{0}\right)_{\text {sing }}\right)$, let $U=\mathcal{M}-X$ let $U_{1}=\mathcal{M}-\pi_{1}\left(\left(T_{0}\right)_{\text {sing }}\right)$ and $U_{2}=\mathcal{M}-\pi_{1}(D)$ so that $U=U_{1} \cup U_{2}$. By Lemma 3.4.1, $X$ is a codimension two subset of $\mathcal{M}$ and it will suffice to prove the claim in $U$. On $U_{2}$, the claim is obvious since $\hat{\nu}_{*} \mathbb{G}_{m, \hat{T}_{0}}\left|U_{2}=\hat{\nu}_{*} \mathcal{B}_{\hat{D}}\right| U_{2}$. So we may assume that $x \in U_{1}$. Let $\Omega$ be an étale open neighborhood of $x$ in $U_{1}$, let $s \in \pi_{1 *} \operatorname{Aut}^{\prime} \mathcal{V}(\Omega)$, and let $f_{s}$ be the corresponding function on $\Omega \times_{\mathcal{M}} \hat{T}_{0}$. If $\tilde{x} \in \Omega \times{ }_{\mathcal{M}} \hat{D}$ lies over $x$, we must show that $f_{s}(\tilde{x})=1$.

For $x \in U_{1}$, let $V_{x}$ be the restriction of $\mathcal{V}$ to the slice $\{x\} \times E$, and similarly for $\bar{V}_{x}$. By the general theory of [11], $\bar{V}_{x}$ is a regular bundle and hence, if $\nu^{-1}(x)=\left\{y_{1}, \ldots, y_{n}\right\}$ where the $y_{i}$ are distinct, then $\bar{V}_{x} \cong \bigoplus_{i=1}^{n}\left(I_{n_{i}} \otimes \lambda_{i}\right)=\bigoplus_{i=1}^{n} \bar{V}_{y_{i}}$, where the $\lambda_{i}$ are line bundles on $E$, pairwise distinct, and indexed by the $y_{i}$, and $I_{n}$ is the unique indecomposable rank $n$ vector bundle on $E$ with a filtration whose successive quotients are all isomorphic to $\mathcal{O}_{E}$. Correspondingly, the semilocal ring $H^{0}\left(\nu^{-1}(x) ; \mathcal{O}_{T_{0}} \mid \nu^{-1}(x)\right)$ is a direct sum of local rings $\bigoplus_{i} R_{i}$, where $R_{i} \cong \mathbb{C}[t] /\left(t^{n_{i}}\right)$ is the local ring of the fiber $\nu^{-1}(x)$ at $y_{i}$. Thus the group of units $R_{i}^{*}$ of $R_{i}$ is canonically isomorphic to $\mathbb{C}^{*} \times S_{i}$, where $S_{i}$ is unipotent and the projection $R_{i}^{*} \rightarrow \mathbb{C}^{*}$ is the evaluation of an invertible function on $\operatorname{Spec} R_{i}$ at the closed point. The 
$\operatorname{ring} R_{i}$ is canonically identified with $\operatorname{Hom}\left(\bar{V}_{y_{i}}, \bar{V}_{y_{i}}\right)$. Using this identification, the filtration on $\bar{V}_{y_{i}}$ is defined by $t^{k} \bar{V}_{y_{i}}$ and the action of $z \in \mathbb{C}^{*}$ is given by multiplication by $z$.

By definition, $y_{i} \in \hat{D}$ if and only if $\lambda_{i}=\mathcal{O}_{E}$. In this case, by the local calculations of [10, Theorem 2.4.3], at a generic point of $D$, the summand of $V_{x}$ corresponding to the trivial line bundle $\mathcal{O}_{E}$ is of the form $\mathcal{O}_{E}^{m_{0}-1} \oplus I_{3}$, and there is a natural exact sequence

$$
0 \rightarrow \mathcal{O}_{E}^{m_{0}} \rightarrow \mathcal{O}_{E}^{m_{0}-1} \oplus I_{3} \rightarrow I_{2} \rightarrow 0
$$

such that the quotient $I_{2}$ is the summand $\bar{V}_{y_{i}}$ of $\bar{V}_{x}$. Let $a$ be the automorphism of $V_{x}$ induced by the section $s \in \pi_{1 *} \operatorname{Aut}^{\prime} \mathcal{V}(\Omega)$ and the choice of the element $\tilde{x} \in \Omega$ lying over $x$. By definition, $a$ preserves the subbundle $\mathcal{O}_{E}^{m_{0}}$ and is the identity on it, and we need to show that $a$ acts trivially on the associated graded of the quotient $I_{2}$. But we can write $a=\operatorname{Id}+b_{1}+b_{2}$, where $b_{1}: I_{3} \rightarrow I_{3}$ is multiplication by a scalar $z$ and $b_{3}$ is nilpotent, and the induced action on the associated graded of $I_{2}$ is multiplication by $1+z$. Restricting to $\mathcal{O}_{E}^{m_{0}}$, we see that $z=0$, and hence $a$ induces the constant function 1 , as claimed. Thus, $f_{s}$ is identically 1 on $\Omega \times_{\mathcal{M}} \hat{D}$.

Returning to the proof of Lemma 3.4.4, we have constructed homomorphisms in both directions between $\pi_{1 *}$ Aut $^{\prime} \mathcal{V}$ and $\hat{\nu}_{*} \mathcal{B}_{\hat{D}}$ which are inverse to each other on a dense open set. Thus, since both sheaves are torsion free, these homomorphisms induce isomorphisms between $\pi_{1 *} \operatorname{Aut}^{\prime} \mathcal{V}$ and $\hat{\nu}_{*} \mathcal{B}_{\hat{D}}$.

Corollary 3.4.6. The composition $\overline{\mathcal{A}}_{\rho} \rightarrow \pi_{1 *} \operatorname{Aut}^{\prime} \mathcal{V} \rightarrow \pi_{1 *}$ Aut $\overline{\mathcal{V}} \cong \hat{\nu}_{*} \mathbb{G}_{m, \hat{T}_{0}}$ defines an embedding of $\overline{\mathcal{A}}_{\rho}$ as a closed subsheaf of $\hat{\nu}_{*} \mathcal{B}_{\hat{D}}$.

Proof. This follows from Proposition 3.1.5 and the previous result, using the fact that $\overline{\mathcal{A}}_{\rho}$ is closed in $\pi_{1 *}$ Aut $\mathcal{V}$ and hence in $\pi_{1 *} \operatorname{Aut}^{\prime} \mathcal{V}$

\subsection{Some auxiliary cohomology computations}

We collect some of the general cohomological results needed to compute $H^{0}(\mathcal{M} ; \mathcal{A})$ and $H^{1}(\mathcal{M} ; \mathcal{A})$. These computations will ultimately reduce to calculations for $H^{i}\left(\mathcal{M} ; \hat{\nu}_{*} \mathbb{G}_{m, \hat{T}_{0}}\right)$ and $H^{i}\left(\mathcal{M} ; \hat{\nu}_{*} \mathcal{B}_{\hat{D}}\right), i=0,1$, or related sheaves, and so we analyze these cohomology groups here.

Lemma 3.5.1. Suppose that $\rho$ is minuscule. Then

$$
H^{0}\left(\mathcal{M} ; \hat{\nu}_{*} \mathbb{G}_{m, \hat{T}_{0}}\right)= \begin{cases}\mathbb{C}^{*}, & \text { if } E \text { is cuspidal } \\ \mathbb{C}^{*} \times \mathbb{Z}, & \text { if } E \text { is nodal. }\end{cases}
$$

In both cases, $H^{1}\left(\mathcal{M} ; \hat{\nu}_{*} \mathbb{G}_{m, \hat{T}_{0}}\right)=\{1\}$. 
Proof. Since $\hat{\nu}$ is finite, $R^{i} \hat{\nu}_{*} \mathcal{S}=0$ for every sheaf $\mathcal{S}$ on $\hat{T}_{0}$, by e.g. [3, Prop. (3.6), p. 24] or [6, Corollary 3.4 , p. 32]. Thus, by the Leray spectral sequence, $H^{i}\left(\mathcal{M} ; \hat{\nu}_{*} \mathbb{G}_{m, \hat{T}_{0}}\right)=$ $H^{i}\left(\hat{T}_{0} ; \mathbb{G}_{m, \hat{T}_{0}}\right)$ for all $i$. For $i=0, H^{0}\left(\hat{T}_{0} ; \mathbb{G}_{m, \hat{T}_{0}}\right)$ is the group of morphisms from $\hat{T}_{0}$ to $\mathbb{G}_{m}$, and for $i=1, H^{1}\left(\hat{T}_{0} ; \mathbb{G}_{m, \hat{T}_{0}}\right)=\operatorname{Pic} \hat{T}_{0}$.

If $E$ is cuspidal, $\hat{T}_{0}=\mathfrak{h} / W_{0}$. Since $W_{0}$ is generated by the reflections in the coroots annihilated by $\varpi, \hat{T}_{0} \cong \mathbb{A}^{r}$. The result is immediate in this case.

Now suppose that $E$ is nodal. Since $\rho$ is minuscule, $\varpi$ is a fundamental weight and hence annihilates all the simple roots except for one, say $\alpha$. Thus, $W_{0}$ is the Weyl group of a subroot system $R_{0}$ of $R$ which is generated by all the simple roots of $R$ except $\alpha$. Clearly $\Lambda_{0}$, the kernel of $\varpi$ on $\Lambda$, is exactly the coroot lattice for $R_{0}$. It follows that $\mathcal{M}_{0}=\left(E_{\text {reg }} \otimes \Lambda_{0}\right) / W_{0} \cong \mathbb{A}^{r-1}$. By Lemma 3.3.1, after an étale base change, the morphism $\hat{T}_{0} \rightarrow E_{\text {reg }}$ becomes a product $\mathcal{M}_{0} \times C$, where $C \cong E_{\text {reg }}$ is a finite étale cover of $E_{\text {reg. }}$. By Lemma 1.2.1, $H^{0}\left(\hat{T}_{0} ; \mathbb{G}_{m, \hat{T}_{0}}\right)=\mathbb{C}^{*} \times \mathbf{X}\left(\mathbb{G}_{m}\right)=\mathbb{C}^{*} \times \mathbb{Z}$ and $H^{1}\left(\hat{T}_{0} ; \mathbb{G}_{m, \hat{T}_{0}}\right)=\operatorname{Pic} \hat{T}_{0}=\{1\}$.

Lemma 3.5.2. Suppose that $\rho$ is properly quasi-minuscule. Then

$$
H^{0}\left(\mathcal{M} ; \hat{\nu}_{*} \mathcal{B}_{\hat{D}}\right)= \begin{cases}\{1\}, & \text { if } E \text { is cuspidal } ; \\ \mathbb{Z}=\left\langle f_{\varpi}\right\rangle, & \text { if } E \text { is nodal. }\end{cases}
$$

In both cases $H^{1}\left(\mathcal{M} ; \hat{\nu}_{*} \mathcal{B}_{\hat{D}}\right)=\{1\}$.

Proof. As in the proof of Lemma 3.5.1, an argument using the Leray spectral sequence shows that it suffices to compute $H^{i}\left(\hat{T}_{0} ; \mathcal{B}_{\hat{D}}\right)$. We have a short exact sequence of sheaves

$$
\{1\} \rightarrow \mathcal{B}_{\hat{D}} \rightarrow \mathbb{G}_{m, \hat{T}_{0}} \rightarrow i_{*} \mathbb{G}_{m, \hat{D}} \rightarrow\{1\}
$$

Again we consider the case when $E$ is cuspidal first. In this case $\hat{T}_{0}$ is again of the form $\mathfrak{h} / W_{0}$ where $W_{0}$ is a group generated by reflections. Thus $\hat{T}_{0}$ is isomorphic to $\mathbb{A}^{r}$. Moreover, $\hat{D}=f_{\varpi}^{-1}(1) \cong \mathcal{M}_{0}$ is isomorphic to $\mathbb{A}^{r-1}$. Thus $H^{0}\left(\hat{D} ; \mathbb{G}_{m}\right) \cong \mathbb{C}^{*}$. Applying the long exact cohomology sequence to the defining exact sequence for $\mathcal{B}_{\hat{D}}$ then establishes the lemma in this case.

Now suppose that $E$ is nodal. Write $\hat{T}_{0}=H / W_{0}$ and $\hat{D}=H_{0} / W_{0}$, where $H_{0}=$ $\mathbb{G}_{m} \otimes \Lambda_{0} \subseteq H=\mathbb{G}_{m} \otimes \Lambda$, and the embedding of $\hat{D}$ in $\hat{T}_{0}$ is induced by the inclusion $H_{0} \subseteq H$. Clearly,

$$
H^{0}\left(H / W_{0} ; \mathbb{G}_{m}\right)=H^{0}\left(H ; \mathbb{G}_{m}\right)^{W_{0}}=\left(\mathbb{C}^{*} \times \mathbf{X}(H)\right)^{W_{0}}=\mathbb{C}^{*} \times \mathbf{X}(H)^{W_{0}} .
$$

Similarly, $H^{0}\left(H_{0} / W_{0} ; \mathbb{G}_{m}\right) \cong \mathbb{C}^{*} \times \mathbf{X}\left(H_{0}\right)^{W_{0}}$, and the restriction map is the identity on the $\mathbb{C}^{*}$ factors and is induced by restriction of characters on the first factor. We claim that the homomorphism $\mathbf{X}(H)^{W_{0}} \rightarrow \mathbf{X}\left(H_{0}\right)^{W_{0}}$ is surjective with kernel $\left\langle f_{\varpi}\right\rangle$. The exact sequence of tori

$$
\{1\} \rightarrow H_{0} \rightarrow H \stackrel{\varpi}{\rightarrow} \mathbb{G}_{m} \rightarrow\{1\}
$$


induces an exact sequence on character groups

$$
\{1\} \rightarrow \mathrm{X}\left(\mathbb{G}_{m}\right)=\mathbb{Z} \rightarrow \mathrm{X}(H) \rightarrow \mathrm{X}\left(H_{0}\right) \rightarrow\{1\},
$$

where the first inclusion is via $\langle\varpi\rangle$. Taking invariants under $W_{0}$, we obtain the sequence

$$
\{1\} \rightarrow\left\langle f_{\varpi}\right\rangle \rightarrow \mathrm{X}(H)^{W_{0}} \rightarrow \mathrm{X}\left(H_{0}\right)^{W_{0}} \rightarrow H^{1}\left(W_{0} ; \mathbb{Z}\right),
$$

where $H^{1}\left(W_{0} ; \mathbb{Z}\right)$ is the group cohomology of $W_{0}$ acting trivially on $\mathbb{Z}$. Since every homomorphism from $W_{0}$ to $\mathbb{Z}$ is zero, $\mathbf{X}(H)^{W_{0}} \rightarrow \mathbf{X}\left(H_{0}\right)^{W_{0}}$ is surjective with kernel $\left\langle f_{\varpi}\right\rangle$ as claimed. The result now follows by considering the long exact cohomology sequence associated to the defining exact sequence for $\mathcal{B}_{\hat{D}}$ as before.

We will need to consider one more computation related to the existence of an involution on $\hat{T}_{0}$. Suppose that the weights of $\rho$ are invariant under multiplication by -1 . Equivalently, $\rho$ is isomorphic to its dual, and it follows that there is a $G$-invariant nondegenerate bilinear form on $V$, necessarily either symmetric or skew-symmetric. In this case, multiplication by -1 on $E_{\text {reg }}$ induces an involution of $\mathcal{M} \times E_{\text {reg }}$ which leaves $T_{0}$ invariant and lifts to the normalization $\hat{T}_{0}$. We denote the corresponding involution of $\hat{T}_{0}$ by $\tau$. In terms of the Weyl group, let $W_{1}$ be the stabilizer of the pair $\{ \pm \varpi\}$ and let $w \in W_{1}$ be an element such that $w(\varpi)=-\varpi$. Then $w$ acts on $\hat{T}_{0}$ as the involution $\tau$. Let $\hat{S}=\hat{T}_{0} /\langle\tau\rangle=\widetilde{\mathcal{M}} / W_{1}$ and let $\varphi: \hat{S} \rightarrow \mathcal{M}$ be the induced morphism. Finally, we set

$$
\left(\hat{\nu}_{*} \mathbb{G}_{m, \hat{T}_{0}}\right)^{-}=\left\{f \in \hat{\nu}_{*} \mathbb{G}_{m, \hat{T}_{0}}: \tau^{*} f=f^{-1}\right\}
$$

in the appropriate sense. Equivalently, we have the norm homomorphism $N: \hat{\nu}_{*} \mathbb{G}_{m, \hat{T}_{0}} \rightarrow$ $\varphi_{*} \mathbb{G}_{m, \hat{S}}$, and by definition $\left(\hat{\nu}_{*} \mathbb{G}_{m, \hat{T}_{0}}\right)^{-}=\operatorname{Ker} N$. If $f$ is the pullback of a function $g$ on $\hat{S}, N(f)=g^{2}$ so that the image of $N$ contains the image of $\varphi_{*} \mathbb{G}_{m, \hat{S}}$ under the squaring homomorphism. Thus $N$ is surjective in the étale topology, and there is an exact sequence

$$
\{1\} \rightarrow\left(\hat{\nu}_{*} \mathbb{G}_{m, \hat{T}_{0}}\right)^{-} \rightarrow \hat{\nu}_{*} \mathbb{G}_{m, \hat{T}_{0}} \rightarrow \varphi_{*} \mathbb{G}_{m, \hat{S}} \rightarrow\{1\}
$$

Clearly, $\left(\hat{\nu}_{*} \mathbb{G}_{m, \hat{T}_{0}}\right)^{-}$is a closed subsheaf of $\hat{\nu}_{*} \mathbb{G}_{m, \hat{T}_{0}}$.

If $\rho$ is properly quasi-minuscule, then the weights of $\rho$ are always invariant under -1 . Clearly $w\left(f_{\varpi}\right)=f_{\varpi}^{-1}$, and so $\hat{D}=f_{\varpi}^{-1}(1)$ is invariant under $\tau$. In fact, since $\varpi$ is a root, the reflection $r_{\varpi}$ in $\varpi$ is an element of $W$ sending $\varpi$ to $-\varpi$ and fixing $\hat{D}=\left(E_{\text {reg }} \otimes(\operatorname{Ker} \varpi)\right) / W_{0}$ pointwise. Let $\left(\hat{\nu}_{*} \mathcal{B}_{\hat{D}}\right)^{-}=\hat{\nu}_{*} \mathcal{B}_{\hat{D}} \cap\left(\hat{\nu}_{*} \mathbb{G}_{m, \hat{T}_{0}}\right)^{-}$. Since $\tau \mid \hat{D}$ is trivial, a function $f$ on $\hat{D}$ such that $\tau^{*} f=f^{-1}$ is necessarily \pm 1 . Since there is also an inclusion of $\{ \pm 1\}$ in $\left(\hat{\nu}_{*} \mathbb{G}_{m, \hat{T}_{0}}\right)^{-}$, it follows that

$$
\left(\hat{\nu}_{*} \mathbb{G}_{m, \hat{T}_{0}}\right)^{-} \cong\left(\hat{\nu}_{*} \mathcal{B}_{\hat{D}}\right)^{-} \times\{ \pm 1\} .
$$

Since $\left(\hat{\nu}_{*} \mathbb{G}_{m, \hat{T}_{0}}\right)^{-}$is a closed subsheaf of $\hat{\nu}_{*} \mathbb{G}_{m, \hat{T}_{0}},\left(\hat{\nu}_{*} \mathcal{B}_{\hat{D}}\right)^{-}$is a closed subsheaf of $\hat{\nu}_{*} \mathcal{B}_{\hat{D}}$. 
Lemma 3.5.3. In the above notation,

$$
H^{0}\left(\mathcal{M} ;\left(\hat{\nu}_{*} \mathbb{G}_{m, \hat{T}_{0}}\right)^{-}\right)= \begin{cases}\{ \pm 1\}, & \text { if } E \text { is cuspidal } \\ \{ \pm 1\} \times \mathbb{Z}, & \text { if } E \text { is nodal. }\end{cases}
$$

Moreover, in both cases $H^{1}\left(\mathcal{M} ;\left(\hat{\nu}_{*} \mathbb{G}_{m, \hat{T}_{0}}\right)^{-}\right)=\{1\}$.

Proof. Using the defining exact sequence for $\left(\hat{\nu}_{*} \mathbb{G}_{m, \hat{T}_{0}}\right)^{-}$and Lemma 3.5.1, it suffices to compute the kernel and cokernel of the norm homomorphism

$$
H^{0}\left(\mathcal{M} ; \hat{\nu}_{*} \mathbb{G}_{m, \hat{T}_{0}}\right) \rightarrow H^{0}\left(\mathcal{M} ; \varphi_{*} \mathbb{G}_{m, \hat{S}}\right)
$$

Clearly $H^{0}\left(\mathcal{M} ; \varphi_{*} \mathbb{G}_{m, \hat{S}}\right)=H^{0}\left(\hat{S} ; \mathbb{G}_{m, \hat{S}}\right)$ is the group of $\tau$-invariant morphisms from $\hat{T}_{0}$ to $\mathbb{G}_{m}$. Thus, if $E$ is cuspidal, the norm map on $H^{0}$ is just $\mathbb{C}^{*} \rightarrow \mathbb{C}^{*}$ given by squaring, so that its kernel is $\{ \pm 1\}$ and its cokernel is trivial. In the nodal case, $H^{0}\left(\hat{T}_{0} ; \mathbb{G}_{m}\right) \cong \mathbb{C}^{*} \times \mathbb{Z}$, where we can take $f_{\varpi}$ to be a generator for $\mathbb{Z}$. The involution $\tau$ acts trivially on the $\mathbb{C}^{*}$ factor and via -1 on the $\mathbb{Z}$ factor, so that $H^{0}\left(\hat{S} ; \mathbb{G}_{m, \hat{S}}\right) \cong \mathbb{C}^{*}$ and the norm map $\mathbb{C}^{*} \times \mathbb{Z}$ is squaring on the first factor and zero on the second. Thus, in this case, the kernel is $\{ \pm 1\} \times \mathbb{Z}$ and the cokernel is trivial.

Using the isomorphism $\left(\hat{\nu}_{*} \mathbb{G}_{m, \hat{T}_{0}}\right)^{-} \cong\left(\hat{\nu}_{*} \mathcal{B}_{\hat{D}}\right)^{-} \times\{ \pm 1\}$ gives:

Lemma 3.5.4. Suppose that $\rho$ is properly quasi-minuscule. Then the weights of $\rho$ are invariant under -1 and $\hat{D} \subset \hat{T}_{0}$ is non-empty and is invariant under -1 . Moreover

$$
H^{0}\left(\mathcal{M} ;\left(\hat{\nu}_{*} \mathcal{B}_{\hat{D}}\right)^{-}\right)= \begin{cases}\{1\}, & \text { if } E \text { is cuspidal } \\ \mathbb{Z}=\left\langle f_{\varpi}\right\rangle, & \text { if } E \text { is nodal. }\end{cases}
$$

In both cases $H^{1}\left(\mathcal{M} ;\left(\hat{\nu}_{*} \mathcal{B}_{\hat{D}}\right)^{-}\right)=\{1\}$.

\section{Explicit examples}

In this section, we give a concrete description of the sheaf $\overline{\mathcal{A}}_{\rho}$ in case $\rho$ is the standard representation of $S L(n+1, \mathbb{C}), S p(2 n, \mathbb{C})$, or $\operatorname{Spin}(n, \mathbb{C})$ or one of the minuscule representations of a group of type $E_{6}$ or $E_{7}$. These cases cover every group which has a minuscule representation, and all of the minuscule representations with the exception of the higher exterior powers of the standard representation of $S L(n+1, \mathbb{C})$ and the spin or half spin representations for $\operatorname{Spin}(n, \mathbb{C})$. In the next section, we will give a general procedure for dealing with all of the properly quasi-minuscule representations and thus give a uniform proof of Thereom 2.2.3 for all groups.

We consider the various cases individually. 


\subsection{Type $A_{n}: G=S L(n+1, \mathbb{C})$}

Let $\rho: S L(n+1, \mathbb{C}) \rightarrow G L(n+1, \mathbb{C})$ be the defining (minuscule) representation and let $\mathcal{V}$ be the corresponding vector bundle over $\mathcal{M} \times E$. The restriction $\mathcal{V} \mid(\{x\} \times E)$ is regular for every $x \in \mathcal{M}$. It follows that $T=T_{0}=\hat{T}_{0}$. Since $\rho$ is faithful, $\overline{\mathcal{A}}_{\rho}=\mathcal{A}$.

Proposition 4.1.1. $\mathcal{A} \subseteq \nu_{*} \mathbb{G}_{m, T}$ is the kernel of the norm map $N: \nu_{*} \mathbb{G}_{m, T} \rightarrow \mathbb{G}_{m, \mathcal{M}}$.

Proof. This is immediate from the identification of $N$ with the determinant homomorphism.

Corollary 4.1.2. There is an exact sequence

$$
\{1\} \rightarrow \mathcal{A} \rightarrow \nu_{*} \mathbb{G}_{m, T} \stackrel{N}{\rightarrow} \mathbb{G}_{m, \mathcal{M}} \rightarrow\{1\}
$$

Proof. It suffices to observe that the image of $N$ contains the $(n+1)^{\text {st }}$ powers of functions in $\mathbb{G}_{m, \mathcal{M}}$ and hence $N$ is surjective in the étale topology.

Thus, there is a exact sequence

$$
0 \rightarrow H^{0}(\mathcal{M} ; \mathcal{A}) \rightarrow H^{0}\left(T ; \mathbb{G}_{m, T}\right) \rightarrow H^{0}\left(\mathcal{M} ; \mathbb{G}_{m, \mathcal{M}}\right) \rightarrow H^{1}(\mathcal{M} ; \mathcal{A}) \rightarrow \operatorname{Pic} T \rightarrow \operatorname{Pic} \mathcal{M} \rightarrow 0
$$

Since $\mathcal{M} \cong \mathbb{A}^{n}, H^{0}\left(\mathcal{M} ; \mathbb{G}_{m, \mathcal{M}}\right)=\mathbb{C}^{*}$. By Lemma 3.5.1 (or directly), Pic $T=0$, and $H^{0}\left(T ; \mathbb{G}_{m, T}\right) \cong \mathbb{C}^{*}$, in the cuspidal case, and $H^{0}\left(T ; \mathbb{G}_{m, T}\right) \cong \mathbb{C}^{*} \times \mathbb{Z}$ in the nodal case. The norm map $N$ is raising to the $(n+1)^{\text {st }}$ power on the $\mathbb{C}^{*}$ factors and is trivial on the $\mathbb{Z}$ factor, if it is present (because $N\left(f_{\varpi}\right)=$ det). Hence $N$ is surjective, so that $H^{1}(\mathcal{M} ; \mathcal{A})=\{1\}$, and $H^{0}(\mathcal{M} ; \mathcal{A})=\operatorname{Ker} N$ is either $\mathbb{Z} /(n+1) \mathbb{Z}$ or $(\mathbb{Z} /(n+1) \mathbb{Z}) \times \mathbb{Z}$, depending on whether $E$ is cuspidal or nodal.

Remark 4.1.3. More generally, let $E$ be any Weierstrass cubic, let $B$ be an arbitrary scheme, and let $\mathcal{V} \rightarrow B \times E$ be a family of regular semistable bundles of rank $n+1$ with trivial determinant. In case $E$ is singular, we do not require that $\mathcal{V} \mid\{x\} \times E$ pulls back to the trivial vector bundle on $\widetilde{E}$ for every $x \in B$. Let $\nu: T_{\mathcal{V}} \rightarrow B$ be the spectral cover; it is compatible with base change. In this case, if $\Xi_{\mathcal{V}}$ is the associated $S L(n+1, \mathbb{C})$-bundle, then arguments as above show that there is an exact sequence

$$
\{1\} \rightarrow \pi_{1 *} \text { Aut } \Xi_{\mathcal{V}} \rightarrow \nu_{*} \mathbb{G}_{m, T_{\mathcal{V}}} \stackrel{N}{\rightarrow} \mathbb{G}_{m, B} \rightarrow\{1\}
$$

A similar remark holds for elliptic fibrations. 


\subsection{Type $C_{n}: G=S p(2 n, \mathbb{C})$}

Let $\rho: G \rightarrow G L(2 n, \mathbb{C})$ be the standard representation. Thus $\rho$ is injective and minuscule. In particular $\overline{\mathcal{A}}_{\rho}=\mathcal{A}$. The corresponding vector bundle $\mathcal{V}$ has regular restriction to each slice $\{x\} \times E$ since the difference of any two distinct weights is a root. Hence $T=T_{0}=\hat{T}_{0}$ in this case.

There is a nondegenerate symplectic form $B$ on $\mathcal{V}$, and hence an isomorphism $\varphi: \mathcal{V} \rightarrow \mathcal{V}^{*}$ via $\varphi(v)(w)=B(v, w)$. Note that $A$ is symplectic if and only if $\varphi \circ A^{-1}=A^{*} \circ \varphi$. As we have seen, the isomorphism $\varphi$ induces an involution $\tau$ on $T=\hat{T}_{0}$ covering the identity on $\mathcal{M}$. We can see $\tau$ quite concretely as follows: we have $T=\operatorname{Spec} \pi_{1 *} \operatorname{Hom}(\mathcal{V}, \mathcal{V})$, viewed as a sheaf of $\mathcal{O}_{\mathcal{M}}$-algebras, and the involution is the one defined by

$$
\tau(A)=\varphi^{-1} \circ A^{*} \circ \varphi .
$$

It is easy to check that this agrees with the involution $\tau$ on $T$ as defined previously. In particular, $A$ is symplectic if and only if $\tau(A)=A^{-1}$. By definition, we have:

Proposition 4.2.1. $\mathcal{A}=\left(\nu_{*} \mathbb{G}_{m, T}\right)^{-}$.

Applying Lemma 3.5 .3 then gives:

Corollary 4.2.2. $H^{1}(\mathcal{M} ; \mathcal{A})=\{1\}$, and $H^{0}(\mathcal{M} ; \mathcal{A})$ is isomorphic to $\{ \pm 1\}$ if $E$ is cuspidal and $\{ \pm 1\} \times \mathbb{Z}$ if $E$ is nodal.

As in the case of $S L(n+1, \mathbb{C})$, there is a relative version of the sheaf $\mathcal{A}$, including the case where $E$ is smooth or certain cases where the $G$-bundles $\Xi \mid\{x\} \times E$ need not pull back to the trivial bundle on the normalization.

\subsection{Type $D_{n}: G=\operatorname{Spin}(2 n, \mathbb{C})$}

In this case, let $\rho$ be the $2 n$-dimensional orthogonal representation, i.e. $\rho$ is the composition $\operatorname{Spin}(2 n, \mathbb{C}) \rightarrow S O(2 n, \mathbb{C}) \rightarrow G L(2 n, \mathbb{C})$. In this case the kernel of $\rho$ is a subgroup of order 2. The representation $\rho$ is minuscule, so that $T=T_{0}$. However, since there are pairs of weights whose difference is not a multiple of a root, there exist $x \in \mathcal{M}$ such that the restriction $\mathcal{V} \mid(\{x\} \times E)$ is not regular. Thus $T_{0}$ is not normal, and hence $\hat{T}_{0} \neq T_{0}$.

As in the symplectic case, the quadratic form on $\mathcal{V}$ defines an isomorphism $\varphi: \mathcal{V} \rightarrow \mathcal{V}^{*}$ The involution $\tau: \hat{T}_{0} \rightarrow \hat{T}_{0}$ is clearly induced by the involution of $\pi_{1 *} \operatorname{Hom}(\mathcal{V}, \mathcal{V})$ defined by $A \mapsto \varphi^{-1} \circ A^{*} \circ \varphi$. Moreover, $A$ is orthogonal if and only if $\tau(A)=A^{-1}$. Thus, via Corollary 3.3.5,

Proposition 4.3.1. $\overline{\mathcal{A}}_{\rho}=\left(\hat{\nu}_{*} \mathbb{G}_{m, \hat{T}_{0}}\right)^{-}$.

Corollary 4.3.2. $H^{1}(\mathcal{M} ; \mathcal{A})=\{1\}$, and $H^{0}(\mathcal{M} ; \mathcal{A})$ is isomorphic to $Z(G)$ if $E$ is cuspidal and $Z(G) \times \mathbb{Z}$ if $E$ is nodal. 
Proof. By Lemma 3.5.3, $H^{1}\left(\mathcal{M} ; \overline{\mathcal{A}}_{\rho}\right)=\{1\}$, and $H^{0}\left(\mathcal{M} ; \overline{\mathcal{A}}_{\rho}\right)$ is either $\{ \pm 1\}$ if $E$ is cuspidal or $\mathbb{Z} \times\{ \pm 1\}$ if $E$ is nodal. By Lemma 3.1.4, $H^{1}(\mathcal{M} ; \mathcal{A})=\{1\}$ and there is an exact sequence

$$
0 \rightarrow \mathbb{Z} / 2 \mathbb{Z} \rightarrow H^{0}(\mathcal{M} ; \mathcal{A}) \rightarrow H^{0}\left(\mathcal{M} ; \overline{\mathcal{A}}_{\rho}\right) \rightarrow\{1\}
$$

It follows that the torsion subgroup of $H^{0}(\mathcal{M} ; \mathcal{A})$ has order 4 and its rank is zero if $E$ is cuspidal and one if $E$ is nodal. Since $H^{0}(\mathcal{M} ; \mathcal{A})$ contains a subgroup isomorphic to $Z(G)$, it follows that $H^{0}(\mathcal{M} ; \mathcal{A})$ is as claimed.

\subsection{Type $B_{n}: G=\operatorname{Spin}(2 n+1, \mathbb{C})$}

In this case, let $\rho$ be the standard representation induced by $\operatorname{Spin}(2 n+1) \rightarrow S O(2 n+1, \mathbb{C}) \subseteq$ $G L(2 n+1, \mathbb{C})$. The kernel of $\rho$ has order two. The representation $\rho$ is properly quasiminuscule and the trivial weight has multiplicity one. The spectral cover is $T=T_{0} \cup T_{0}$, where $T_{0}$ is reduced. It is straightforward to check that, if $E$ is cuspidal, then $T_{0}$ is normal, whereas $T_{0}$ is not normal in case $E$ is nodal. The quadratic form on $\mathcal{V}$ induces an involution on $T_{0}$ and $\hat{T}_{0}$. With this said, we have:

Proposition 4.4.1. $\overline{\mathcal{A}}_{\rho}=\left(\hat{\nu}_{*} \mathcal{B}_{\hat{D}}\right)^{-}$.

Proof. By Corollary 3.4.6, $\overline{\mathcal{A}}_{\rho}$ is a closed subsheaf of $\hat{\nu}_{*} \mathcal{B}_{\hat{D}}$. On the other hand, $\left(\hat{\nu}_{*} \mathcal{B}_{\hat{D}}\right)^{-}$ is also a closed subsheaf of $\hat{\nu}_{*} \mathcal{B}_{\hat{D}}$. Thus, it suffices to prove that the restrictions of $\overline{\mathcal{A}}_{\rho}$ and $\left(\hat{\nu}_{*} \mathcal{B}_{\hat{D}}\right)^{-}$to the étale open set $U$ of Lemma 3.1 .2 and Corollary 3.1.3 agree. On $U$, $\mathcal{V}=\bigoplus_{\mu} \mathcal{L}_{\mu}$, where $\overline{\mathcal{A}}_{\rho}$ acts trivially on $\mathcal{L}_{0}$, acts on $\mathcal{L}_{\mu}$ via the character $\mu$, and hence on $\mathcal{L}_{-\mu}$ via the character $\mu^{-1}$. Clearly, this identifies $\overline{\mathcal{A}}_{\rho} \mid U$ with $\left(\hat{\nu}_{*} \mathcal{B}_{\hat{D}}\right)^{-} \mid U$.

Standard arguments, using Lemma 3.5.4, then show:

Corollary 4.4.2. $H^{1}(\mathcal{M} ; \mathcal{A})=\{1\}$, and $H^{0}(\mathcal{M} ; \mathcal{A})$ is isomorphic to $Z(G)$ if $E$ is cuspidal and $Z(G) \times \mathbb{Z}$ if $E$ is nodal.

\subsection{Type $E_{6}$}

Let $G$ be of type $E_{6}$ and let $\rho: G \rightarrow G L(V)$ be one of the 27-dimensional minuscule representations of $G$. The representation $\rho$ is injective and minuscule. Thus $T=T_{0}$. However, $T_{0}$ is never normal, since there are pairs of weights of $\rho$ whose difference is not a multiple of a root. The group $G$ is the stabilizer of a symmetric cubic form $C$ in the automorphism group of $V$. If we decompose $V$ into a direct sum of weight spaces $V=\bigoplus_{\mu} L_{\mu}$, then the form $C$ preserves the decomposition of $V$ into weight spaces. It is non-trivial on $L_{\mu_{1}} \otimes L_{\mu_{2}} \otimes L_{\mu_{3}}$ if and only if $\mu_{1}+\mu_{2}+\mu_{3}=0$.

Claim 4.5.1. Let $\widetilde{H}$ be the set of $A \in G L(V)$ such that $A \mid L_{\mu}$ is multiplication by a nonzero scalar $a_{\mu}$ and such that the $a_{\mu}$ satisfy: for every triple of weights $\left(\mu_{1}, \mu_{2}, \mu_{3}\right)$ such $\mu_{1}+\mu_{2}+$ $\mu_{3}=0, a_{\mu_{1}} a_{\mu_{2}} a_{\mu_{3}}=1$. Then $\rho(H)=\widetilde{H}$. 
Proof. Clearly, if $h \in H$, then $\rho(h) \mid L_{\mu}$ is multiplication by $a_{\mu}=\mu(h)$ and hence the $a_{\mu}$ satisfy the multiplicative property of the claim. Conversely, if $A \in \widetilde{H}$, then $A$ preserves $C$, so that $A=\rho(g)$ for some $g \in G$. As $A$ commutes with $\rho(H)$ and $\rho$ is faithful, $g$ commutes with $H$ and hence $g \in H$.

We turn now to a description of $\mathcal{A}=\overline{\mathcal{A}}_{\rho}$. By Corollary 3.3.5, $\mathcal{A}$ is a closed subsheaf of $\hat{\nu}_{*} \mathbb{G}_{m, \hat{T}_{0}}$. We will describe this subsheaf by means of a correspondence on $\hat{T}_{0}$. Recall that $W_{0}$ is the stabilizer of the highest weight $\varpi$ of $\rho$.

Lemma 4.5.2. If $\mu_{1}$ is a weight of $\rho$, then there exist weights $\mu_{2}, \mu_{3}$ of $\rho$ such that $\mu_{1}+\mu_{2}+$ $\mu_{3}=0$. The Weyl group $W$ acts transitively on the set of ordered pairs $\left(\left\{\mu_{1}, \mu_{2}, \mu_{3}\right\}, \mu_{1}\right)$, where $\left\{\mu_{1}, \mu_{2}, \mu_{3}\right\}$ is an unordered triple of weights of $\rho$ whose sum is zero.

Proof. Since the Weyl group acts transitively on the weights of $\rho$, it suffices to show that the stabilizer $W_{0}$ of $\varpi$ acts transitively on the set of weights $\mu$ with the property that $\varpi+\mu$ is the negative of a weight of $\rho$. The kernel of $\varpi$ in the coroot lattice of $E_{6}$ is the coroot lattice of $D_{5}$. Thus $W_{0}$ contains a subgroup isomorphic to the Weyl group of $D_{5}$. As a representation of $\operatorname{Spin}(10), V$ is a direct sum $\mathbb{C} \oplus V^{10} \oplus S^{16}$, where $V^{10}$ is the orthogonal representation and $S^{16}$ is one of the half spinor representations. The weight spaces $L_{\mu}$, where $\mu$ has the property that $\varpi+\mu$ is the negative of a weight of $\rho$, are all weight spaces in $V^{10}$. Since the Weyl group of $D_{5}$ acts transitively on these weights, the result follows.

In terms of cubic surfaces, the weights of $\rho$ may be identified with lines on a smooth cubic surface, and the lemma says that the stabilizer $W_{0}$ in $W$ of a line $\ell$ acts transitively on the 10 lines which meet $\ell$.

Given the weight $\varpi$, fix weights $\mu_{2}, \mu_{3}$ such that $\varpi+\mu_{2}+\mu_{3}=0$. Let $W^{\prime}$ be the stabilizer of the unordered triple $\left\{\varpi, \mu_{2}, \mu_{3}\right\}$ and let $W^{\prime \prime}$ be the stabilizer of the ordered pair $\left(\left\{\varpi, \mu_{2}, \mu_{3}\right\}, \varpi\right)$. Thus $W^{\prime \prime}$ is a subgroup both of $W_{0}$ and $W^{\prime}$. Set $S^{\prime}=\widetilde{\mathcal{M}} / W^{\prime}$ and $S^{\prime \prime}=\widetilde{\mathcal{M}} / W^{\prime \prime}$. Thus there is a diagram of schemes

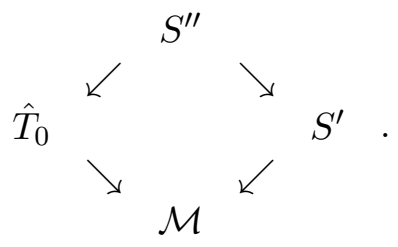

Let $\nu^{\prime}: S^{\prime} \rightarrow \mathcal{M}$ and $\nu^{\prime \prime}: S^{\prime \prime} \rightarrow \mathcal{M}$ be the induced morphisms. Then there is a homomorphism $c: \hat{\nu}_{*} \mathbb{G}_{m, \hat{T}_{0}} \rightarrow \nu_{*}^{\prime} \mathbb{G}_{m, S^{\prime}}$ defined by pulling a function on $\hat{T}_{0}$ back to $S^{\prime \prime}$, followed by the norm homomorphism $\nu_{*}^{\prime \prime} \mathbb{G}_{m, S^{\prime \prime}} \rightarrow \nu_{*}^{\prime} \mathbb{G}_{m, S^{\prime}}$. By Lemma 1.1.2, Ker $c$ is a closed subsheaf of $\hat{\nu}_{*} \mathbb{G}_{m, \hat{T}_{0}}$. If $x \in \mathcal{M}$ is a point such that the morphism $\widetilde{\mathcal{M}} \rightarrow \mathcal{M}$ is étale and $\tilde{x} \in \widetilde{\mathcal{M}}$ lies in the fiber over $x$, the fiber $\nu^{-1}(x)$ is identified with the set of weights of $\rho$ 
and the fiber $\left(\nu^{\prime}\right)^{-1}(x)$ is identified with the set of unordered triples $\left\{\mu_{1}, \mu_{2}, \mu_{3}\right\}$ such that $\mu_{1}+\mu_{2}+\mu_{3}=0$. Clearly,

$$
c(f)\left(\left\{\mu_{1}, \mu_{2}, \mu_{3}\right\}\right)=f\left(\mu_{1}\right) f\left(\mu_{2}\right) f\left(\mu_{3}\right)
$$

and hence $c(f)=1$ if and only if $f\left(\mu_{1}\right) f\left(\mu_{2}\right) f\left(\mu_{3}\right)=1$ for all such triples.

Proposition 4.5.3. $\mathcal{A}=\operatorname{Ker} c$.

Proof. Since both $\mathcal{A}$ and Ker $c$ and closed subsheaves of the torsion free sheaf $\hat{\nu}_{*} \mathbb{G}_{m, \hat{T}_{0}}$, it suffices to check the equality on an étale open dense set. Let $U$ be the étale open set of Lemma 3.1.2 and Corollary 3.1.3. The result over $U$ follows immediately from the above description of Ker $c$ and Claim 4.5.1.

Proposition 4.5.4. $H^{1}(\mathcal{M} ; \mathcal{A})=\{1\}$, and $H^{0}(\mathcal{M} ; \mathcal{A})$ is isomorphic to $\mathbb{Z} / 3 \mathbb{Z}$ if $E$ is cuspidal and $(\mathbb{Z} / 3 \mathbb{Z}) \times \mathbb{Z}$ if $E$ is nodal.

Proof. Let $\mathcal{I}=\operatorname{Im} c$. Then $\mathcal{I}$ is a subsheaf of $\nu_{*}^{\prime} \mathbb{G}_{m, S^{\prime}}$ and there is an exact sequence

$$
\{1\} \rightarrow \mathcal{A} \rightarrow \hat{\nu}_{*} \mathbb{G}_{m, \hat{T}_{0}} \rightarrow \mathcal{I} \rightarrow\{1\}
$$

First suppose that $E$ is cuspidal. Since there is a finite morphism from $\widetilde{\mathcal{M}}=\mathfrak{h}$ to $S^{\prime}$, $\Gamma\left(S^{\prime}, \mathbb{G}_{m, S^{\prime}}\right) \subseteq \Gamma\left(\mathfrak{h}, \mathbb{G}_{m, \mathfrak{h}^{*}}\right)=\mathbb{C}^{*}$ as $\mathfrak{h}$ is affine. Thus $\Gamma\left(S^{\prime}, \mathbb{G}_{m, S^{\prime}}\right)=\mathbb{C}^{*}$ consists of the nonzero constant functions. It follows that the homomorphism $c: H^{0}\left(\mathcal{M} ; \hat{\nu}_{*} \mathbb{G}_{m, \hat{T}_{0}}\right) \rightarrow$ $H^{0}\left(\mathcal{M} ; \nu_{*}^{\prime} \mathbb{G}_{m, S^{\prime}}\right)$ is the cubing homomorphism from $\mathbb{C}^{*}$ to $\mathbb{C}^{*}$ and factors through the subgroup $H^{0}(\mathcal{M} ; \mathcal{I})$ of $H^{0}\left(\mathcal{M} ; \nu_{*}^{\prime} \mathbb{G}_{m, S^{\prime}}\right)$. This forces $H^{0}(\mathcal{M} ; \mathcal{I})=H^{0}\left(\mathcal{M} ; \nu_{*}^{\prime} \mathbb{G}_{m, S^{\prime}}\right)=\mathbb{C}^{*}$, and the induced homomorphism

$$
H^{0}\left(\mathcal{M} ; \hat{\nu}_{*} \mathbb{G}_{m, \hat{T}_{0}}\right) \rightarrow H^{0}(\mathcal{M} ; \mathcal{I})
$$

is again the cubing homomorphism $\mathbb{C}^{*} \rightarrow \mathbb{C}^{*}$. Thus it is surjective with kernel $\mathbb{Z} / 3 \mathbb{Z}$. It follows that $H^{0}(\mathcal{M} ; \mathcal{A})=\mathbb{Z} / 3 \mathbb{Z}$ and that $H^{1}(\mathcal{M} ; \mathcal{A}) \subseteq H^{1}\left(\mathcal{M} ; \hat{\nu}_{*} \mathbb{G}_{m, \hat{T}_{0}}\right)$, which is trivial by Lemma 3.5.1. This concludes the proof in the cuspidal case.

Now assume that $E$ is nodal. By Lemma 3.5.1, $H^{0}\left(\mathcal{M} ; \hat{\nu}_{*} \mathbb{G}_{m, \hat{T}_{0}}\right) \cong \mathbb{C}^{*} \times \mathbb{Z}$. As in the proof of the cuspidal case, it will suffice to prove that $H^{0}(\mathcal{M} ; \mathcal{I})=H^{0}\left(\mathcal{M} ; \nu_{*}^{\prime} \mathbb{G}_{m, S^{\prime}}\right)=\mathbb{C}^{*}$, and that the induced homomorphism $H^{0}\left(\mathcal{M} ; \hat{\nu}_{*} \mathbb{G}_{m, \hat{T}_{0}}\right) \rightarrow H^{0}(\mathcal{M} ; \mathcal{I})$ is the homomorphism from $\mathbb{C}^{*} \times \mathbb{Z}$ to $\mathbb{C}^{*}$ given by $(z, n) \mapsto z^{3}$. Clearly $c\left(f_{\varpi}\right)=1$, so that it suffices to show that $H^{0}\left(\mathcal{M} ; \nu_{*}^{\prime} \mathbb{G}_{m, S^{\prime}}\right)=H^{0}\left(S^{\prime} ; \mathbb{G}_{m, S^{\prime}}\right)=\mathbb{C}^{*}$. As in the proof of Lemma 3.5.2,

$$
H^{0}\left(S^{\prime} ; \mathbb{G}_{m, S^{\prime}}\right)=H^{0}\left(H ; \mathbb{G}_{m, H}\right)^{W^{\prime}}=\left(\mathbb{C}^{*} \times \mathbf{X}(H)\right)^{W^{\prime}}=\mathbb{C}^{*} \times \mathbf{X}(H)^{W^{\prime}} .
$$

Since $\mathbf{X}(H)=\Lambda^{*}$, it suffices to prove that $\left(\mathfrak{h}^{*}\right)^{W^{\prime}}=0$, or equivalently that $\mathfrak{h}^{W^{\prime}}=0$. It is easy to check that, if $\varpi, \mu_{2}, \mu_{3}$ are three weights of $\rho$ whose sum is zero, then $\left\{\varpi, \mu_{2}, \mu_{3}\right\}^{\perp}$ is a root lattice of type $D_{4}$ and hence $W^{\prime}$ contains the Weyl group of $D_{4}$. Also, by Lemma 4.5.2, $W^{\prime}$ acts transitively on the set $\left\{\varpi, \mu_{2}, \mu_{3}\right\}$. From this, it follows that there are no $W^{\prime}-$ invariant vectors in $\mathfrak{h}=\operatorname{span}\left\{\varpi, \mu_{2}, \mu_{3}\right\} \oplus\left\{\varpi, \mu_{2}, \mu_{3}\right\}^{\perp}$.

Thus $H^{0}\left(S^{\prime} ; \mathbb{G}_{m, S^{\prime}}\right)=\mathbb{C}^{*}$, and the rest of the proof proceeds as in the cuspidal case. 


\subsection{Type $E_{7}$}

Let $G$ be of type $E_{7}$, and let $\rho: G \rightarrow G L(V)$ be the 56 -dimensional irreducible representation of $E_{7}$. Since $\rho$ is injective and minuscule, $\overline{\mathcal{A}}_{\rho}=\mathcal{A}$ and $T=T_{0}$. Since there are pairs of weights of $\rho$ whose difference is not a multiple of a root, $T_{0}$ is never normal. The group $G$ is the stabilizer of a non-degenerate skew form and a symmetric quartic form on $V$. Decomposing $V$ as a direct sum of weight spaces, $V=\bigoplus_{\mu} L_{\mu}$, the skew form is non-zero exactly on tensor products of the form $L_{\mu} \otimes L_{-\mu}$ and the quartic form is nontrivial exactly on tensor products of the form $L_{\mu_{1}} \otimes L_{\mu_{2}} \otimes L_{\mu_{3}} \otimes L_{\mu_{4}}$ when $\mu_{1}+\mu_{2}+\mu_{3}+\mu_{4}=0$ and $\mu_{1}+\mu_{i} \neq 0$ for $i=2,3,4$. We say that an unordered quadruple $\left\{\mu_{1}, \mu_{2}, \mu_{3}, \mu_{4}\right\}$ of weights properly sums to zero $\mu_{1}+\mu_{2}+\mu_{3}+\mu_{4}=0$ and $\mu_{i}+\mu_{j} \neq 0$ for all $i, j, 1 \leq i, j \leq 4$.

An argument as for the case of $E_{6}$ then shows:

Claim 4.6.1. Let $\widetilde{H}$ be the set of $A \in G L(V)$ such that $A \mid L_{\mu}$ is multiplication by a scalar $a_{\mu}$ and such that the $a_{\mu}$ satisfy: $a_{\mu} a_{-\mu}=1$ for all weights $\mu$ and $a_{\mu_{1}} a_{\mu_{2}} a_{\mu_{3}} a_{\mu_{4}}=1$ whenever $\left\{\mu_{1}, \mu_{2}, \mu_{3}, \mu_{4}\right\}$ properly sums to zero. Then $\rho(H)=\widetilde{H}$.

We turn now to the description of $\mathcal{A}$. Since the skew form identifies $V$ and $V^{*}$, there is an involution $\tau$ on $\hat{T}_{0}$. By Claim 4.6.1, $\mathcal{A}$ is a closed subsheaf of $\left(\hat{\nu}_{*} \mathbb{G}_{m, \hat{T}_{0}}\right)^{-}$. As in the case of $E_{6}$, we will describe this subsheaf by a correspondence. We begin with a lemma on the Weyl group:

Lemma 4.6.2. The group $W$ acts transitively on the set of all pairs $\left(\left\{\mu_{1}, \mu_{2}, \mu_{3}, \mu_{4}\right\}, \mu_{1}\right)$ consisting of a quadruple which properly sums to zero and one of the weights in the quadruple. Moreover, if $\left\{\varpi, \mu_{2}, \mu_{3}, \mu_{4}\right\}$ is such a quadruple, the stabilizer $W_{0}$ of $\varpi$ contains an element which cyclically permutes the set $\left\{\mu_{2}, \mu_{3}, \mu_{4}\right\}$.

Proof. Since $W$ acts transitively on the weights of $\rho$, to prove the first statement, it suffices to show that $W_{0}$ acts transitively on the set of all quadruples $\left\{\varpi, \mu_{2}, \mu_{3}, \mu_{4}\right\}$ which properly sum to zero. The kernel of $\varpi$ is the coroot lattice $\Lambda_{1}$ of $E_{6}$, and hence $W_{0}$ contains a subgroup $W_{1}$ isomorphic to the Weyl group of $E_{6}$. Let $G_{1}$ be the corresponding subgroup of $G$ of type $E_{6}$. The representation $V$ viewed as a representation of $G_{1}$, is isomorphic to a direct sum $\mathbb{C} \oplus \mathbb{C} \oplus A \oplus \bar{A}$ where $A$ and $\bar{A}$ are the two non-isomorphic minuscule representations of $G_{1}$. The weight spaces $L_{\mu}$ with $\mu$ as above form one of the minuscule factors $A$ or $\bar{A}$. In fact, possibly after relabeling, the quadruples containing $\varpi$ which properly sum to zero consist of $\varpi$ and a triple of weights which become weights for the maximal torus of $G_{1}$ acting on $A$ and which sum to zero as weights acting on $\Lambda_{1}$. Since $W_{1}$ acts transitively on these weights, the first statement follows. The second is a consequence of Lemma 4.5.2.

Fix a quadruple $\left\{\varpi, \mu_{2}, \mu_{3}, \mu_{4}\right\}$ which properly sums to zero. Let $W^{\prime}$ be the stabilizer of $\left\{\varpi, \mu_{2}, \mu_{3}, \mu_{4}\right\}$ and let $W^{\prime \prime}$ be the stabilizer of the pair $\left(\left\{\varpi, \mu_{2}, \mu_{3}, \mu_{4}\right\}, \varpi\right)$. Thus $W^{\prime \prime}$ is a subgroup both of $W_{0}$ and of $W^{\prime}$. As in the case of $E_{6}$, we form the spaces $S^{\prime}=\widetilde{\mathcal{M}} / W^{\prime}$ and $S^{\prime \prime}=\widetilde{\mathcal{M}} / W^{\prime \prime}$. Clearly, the degree of the morphism $S^{\prime \prime} \rightarrow S^{\prime}$ is 4 . If $\nu^{\prime}: S^{\prime} \rightarrow$ 
$\mathcal{M}$ is the induced morphism, then we can again define the homomorphism $c: \hat{\nu}_{*} \mathbb{G}_{m, \hat{T}_{0}} \rightarrow$ $\hat{\nu}_{*}^{\prime} \mathbb{G}_{m, S^{\prime}}$. Clearly, the involution $\tau$ extends to involutions on $S^{\prime \prime}$ and $S^{\prime}$, also denoted $\tau$, and $c$ is equivariant. Thus the restriction of $c$ to $\left(\hat{\nu}_{*} \mathbb{G}_{m, \hat{T}_{0}}\right)^{-}$takes values in $\left(\hat{\nu}_{*}^{\prime} \mathbb{G}_{m, S^{\prime}}\right)^{-}$. An argument as in the proof of Proposition 4.5.3 gives:

Proposition 4.6.3. $\mathcal{A}=\left(\hat{\nu}_{*} \mathbb{G}_{m, \hat{T}_{0}}\right)^{-} \cap \operatorname{Ker} c$.

Proposition 4.6.4. $H^{1}(\mathcal{M} ; \mathcal{A})=\{1\}$, and $H^{0}(\mathcal{M} ; \mathcal{A})$ is isomorphic to $\mathbb{Z} / 2 \mathbb{Z}$ if $E$ is cuspidal and $(\mathbb{Z} / 2 \mathbb{Z}) \times \mathbb{Z}$ if $E$ is nodal.

Proof. Let $\mathcal{I}=\operatorname{Im}\left(c \mid\left(\hat{\nu}_{*} \mathbb{G}_{m, \hat{T}_{0}}\right)^{-}\right)$. Then $\mathcal{I}$ is a subsheaf of $\left(\nu_{*}^{\prime} \mathbb{G}_{m, S^{\prime}}\right)^{-}$and there is an exact sequence

$$
\{1\} \rightarrow \mathcal{A} \rightarrow\left(\hat{\nu}_{*} \mathbb{G}_{m, \hat{T}_{0}}\right)^{-} \rightarrow \mathcal{I} \rightarrow\{1\} .
$$

The proof is completed, using Lemma 3.5.3, by showing that $H^{0}(\mathcal{M} ; \mathcal{I})=0$.

We begin by showing that $H^{0}\left(\mathcal{M} ;\left(\nu_{*}^{\prime} \mathbb{G}_{m, S^{\prime}}\right)^{-}\right)=\{ \pm 1\}$. In the case when $E$ is cuspidal, $S^{\prime}$ is a quotient of $\mathfrak{h}$ by a finite group and hence $H^{0}\left(\mathcal{M} ; \nu_{*}^{\prime} \mathbb{G}_{m, S^{\prime}}\right)=\mathbb{C}^{*}$. Since $\tau$ acts trivially on this $\mathbb{C}^{*}, H^{0}\left(\mathcal{M} ;\left(\nu_{*}^{\prime} \mathbb{G}_{m, S^{\prime}}\right)^{-}\right)=\{ \pm 1\}$. When $E$ is nodal, $H^{0}\left(\mathcal{M} ; \nu_{*}^{\prime} \mathbb{G}_{m, S^{\prime}}\right)$ is equal to the product $\mathbb{C}^{*} \times \mathbf{X}(H)^{W^{\prime}}$ and we must compute the second factor. Given the quadruple $\left\{\varpi, \mu_{2}, \mu_{3}, \mu_{4}\right\}$ which properly sums to zero, let $\Lambda^{\prime}$ be the intersection of the kernels of the weights $\varpi, \mu_{2}, \mu_{3}, \mu_{4}$ and let $H^{\prime} \subseteq H$ be the corresponding closed connected subgroup. Note that $\Lambda^{\prime}$ is a coroot lattice of type $D_{4}$. Let $H^{\prime \prime}$ be the torus whose Lie algebra is spanned by the weights, which we view as elements of $\mathfrak{h}$ via the inner product. Note that $W^{\prime}$ acts on $H^{\prime}$ and $H^{\prime \prime}$, and there is a $W^{\prime}$-equivariant isogeny $H^{\prime} \times H^{\prime \prime} \rightarrow H$. Correspondingly, there is a $W^{\prime}$-equivariant embedding $\mathrm{X}(H) \rightarrow \mathrm{X}\left(H^{\prime}\right) \oplus \mathrm{X}\left(H^{\prime \prime}\right)$. Since $W^{\prime}$ contains a subgroup generated by the reflections in the coroots contained in $\Lambda^{\prime}$, which is the Weyl group of $D_{4}$, it follows that $\mathrm{X}\left(H^{\prime}\right)^{W^{\prime}}=0$. As for the second factor $\mathrm{X}\left(H^{\prime \prime}\right) \cong \mathbb{Z}^{3}$, by Lemma 4.6.2 there is an element of $W^{\prime}$ which fixes $\varpi$ and cyclically permutes $\mu_{2}, \mu_{3}, \mu_{4}$. On the other hand, again by Lemma 4.6.2, there is an element of $W^{\prime}$ which sends $\varpi$ to $\mu_{2}$. These two elements generate a subgroup of the symmetric group $S_{4}$ which is of index at most two and hence contains the alternating group. It follows that $\mathrm{X}\left(H^{\prime \prime}\right)^{W^{\prime}}=0$ as well. Thus again $H^{0}\left(\mathcal{M} ; \nu_{*}^{\prime} \mathbb{G}_{m, S^{\prime}}\right)=\mathbb{C}^{*}$ and $H^{0}\left(\mathcal{M} ;\left(\nu_{*}^{\prime} \mathbb{G}_{m, S^{\prime}}\right)^{-}\right)=\{ \pm 1\}$.

Thus $H^{0}\left(\mathcal{M} ;\left(\nu_{*}^{\prime} \mathbb{G}_{m, S^{\prime}}\right)^{-}\right)=\{ \pm 1\}$, where the functions in question are constant functions on $S^{\prime}$. The final step is to show that the image of $H^{0}(\mathcal{M} ; \mathcal{I})$ in $H^{0}\left(\mathcal{M} ;\left(\nu_{*}^{\prime} \mathbb{G}_{m, S^{\prime}}\right)^{-}\right)=$ $\{ \pm 1\}$ is trivial. Let $x_{0} \in \mathcal{M}$ be the image of the identity in $\widetilde{\mathcal{M}}$. Since the identity is fixed by $W$, there is a unique preimage of $x_{0}$ in $\hat{T}_{0}$, as well as in $S^{\prime}$ and $S^{\prime \prime}$, and all of these points are $\tau$-invariant. Let $U \rightarrow \mathcal{M}$ be an étale neighborhood of $x_{0}$ and $u$ a point in the preimage of $x_{0}$. Then there is a unique point $\hat{u} \in U \times \times_{\mathcal{M}} \hat{T}_{0}$ lying above $u$, and similarly there are unique points $u^{\prime} \in U \times_{\mathcal{M}} S^{\prime}$ and $u^{\prime \prime} \in U \times_{\mathcal{M}} S^{\prime \prime}$ above $u$. In particular, $\tau\left(u^{\prime \prime}\right)=u^{\prime \prime}$, where we continue to denote by $\tau$ the induced involution on $U \times_{\mathcal{M}} S^{\prime \prime}$. Hence, if $f \in\left(\hat{\nu}_{*} \mathbb{G}_{m, \hat{T}_{0}}\right)^{-}(U)$, then $f(\hat{u})= \pm 1$, and the value of the pullback of $f$ to $U \times_{\mathcal{M}} S^{\prime \prime}$ at $u^{\prime \prime}$ is again \pm 1 . Finally, since $u^{\prime \prime}$ is a point of total ramification for the morphism $S^{\prime \prime} \rightarrow S^{\prime}$, and the degree of the morphism is 4 , the value of $c(f)$ at $u^{\prime}$ is $( \pm 1)^{4}=1$. It follows that 
every function in $H^{0}(\mathcal{M} ; \mathcal{I})$ takes the value 1 at $u^{\prime}$, and since these functions are constant, it must be identically 1 . Thus $H^{0}(\mathcal{M} ; \mathcal{I})=\{1\}$ as claimed.

\section{The general case}

Our goal in this section is to give a unified proof of Theorem 2.2.3. Throughout this section, let $\rho: G \rightarrow G L(V)$ denote the unique properly quasi-minuscule representation of $G$. Hence Ker $\rho=Z(G)$ and $\overline{\mathcal{A}}_{\rho}=\mathcal{A} / Z(G)$.

\subsection{Identification of the image torus}

Let $R_{s}$ be the set of short roots of $G$. It is the set of nonzero weights of $\rho$. Let $m_{0} \geq 1$ be the multiplicity of the trivial weight in $\rho$. For $\alpha \in R_{s}$, let $L_{\alpha}$ denote the corresponding one-dimensional weight space of $V$. Let $L_{0}$ be the subspace of $V$ where $H$ acts trivially.

Lemma 5.1.1. $\rho(H)$ is the subgroup $\widetilde{H}$ of $G L(V)$ consisting of the $A$ such that $A \mid L_{0}=\mathrm{Id}$, $A \mid L_{\alpha}$ is multiplication by a nonzero scalar $a_{\alpha}$ and the $a_{\alpha}$ satisfy: $a_{\alpha} a_{-\alpha}=1$, and, for every triple $(\alpha, \beta, \gamma) \in R_{s}^{3}$ such that $\alpha+\beta+\gamma=0, a_{\alpha} a_{\beta} a_{\gamma}=1$.

Proof. The element $\rho(h)$ acts on $L_{0}$ as the identity and on $L_{\alpha}$ via $\alpha(h)$. Thus clearly $\rho(H) \subseteq \widetilde{H}$. To see the opposite inclusion, we begin with the following general discussion. Under the identification of $W / W_{0}$ with $R_{s}$, there is a homomorphism $\varphi: \mathbb{Z}\left[W / W_{0}\right] \rightarrow \Lambda^{*}$. Here, if $e_{\alpha}$ is the basis vector corresponding to $\alpha, \varphi\left(e_{\alpha}\right)=\alpha$. The image of $\varphi$ is the span of the short roots, which is just the root lattice $Q(R)$. The kernel $K$ of $\varphi$ is the set of all relations among the elements of $R_{s}$.

Lemma 5.1.2. The kernel $K$ is generated by the following two types of relations:

(i) For $\alpha \in R_{s}, e_{\alpha}+e_{-\alpha}$;

(ii) For $\alpha, \beta, \gamma \in R_{s}$ such that $\alpha+\beta=\gamma, e_{\alpha}+e_{\beta}-e_{\gamma}$.

Proof. If $G$ is not simply laced, the set $R_{s}$ forms a root system whose span is that of $R$. Thus it suffices to consider the case $R=R_{s}$, i.e. $G$ is simply laced (but not necessarily irreducible). Let $v=\sum_{\alpha \in R_{s}} n_{\alpha} e_{\alpha} \in K$, so that $\sum_{\alpha \in R_{s}} n_{\alpha} \alpha=0$. Using relations of type (i), we may assume that all of the $\alpha$ are positive roots. If $\alpha_{1}, \ldots, \alpha_{r}$ are the simple roots, and $\alpha=\sum_{i} n_{i} \alpha_{i}$ is a positive root, let $\ell(\alpha)=\sum_{i} n_{i}$. The proof is by induction on the largest value of $\ell(\alpha)$ where $n_{\alpha} \neq 0$. If this number is greater than one, then $\alpha=\alpha_{i}+\beta$ for some $i$, where $\ell(\beta)=\ell(\alpha)-1$. Applying the relation $n_{\alpha}\left(e_{\alpha}-e_{\alpha_{i}}-e_{\beta}\right)$ then eliminates $e_{\alpha}$. Eventually we reach the stage where all $\alpha$ are simple. Since the simple roots are linearly independent, it follows that at this stage $v=0$. 
Let $F$ be the free $\mathbb{Z}$-module with basis $f_{\alpha}, \alpha \in R_{s}^{+}$and $f_{\alpha, \beta}$, where $\alpha, \beta$, and $\alpha+\beta \in R_{s}$. Let $\psi: F \rightarrow \mathbb{Z}\left[W / W_{0}\right]$ be defined by $\psi\left(f_{\alpha}\right)=e_{\alpha}+e_{-\alpha}$ and $\psi\left(f_{\alpha, \beta}\right)=e_{\alpha}+e_{\beta}-e_{\alpha+\beta}$. The preceding lemma implies that

$$
F \stackrel{\psi}{\rightarrow} \mathbb{Z}\left[W / W_{0}\right] \stackrel{\varphi}{\rightarrow} Q(R) \rightarrow 0
$$

is exact, where $Q(R)$ is the root lattice.

Applying $\operatorname{Hom}\left(\cdot, \mathbb{C}^{*}\right)$ to this sequence gives an exact sequence of tori:

$$
\{1\} \rightarrow \operatorname{Hom}\left(Q(R), \mathbb{C}^{*}\right) \rightarrow \operatorname{Hom}\left(\mathbb{Z}\left[W / W_{0}\right], \mathbb{C}^{*}\right) \rightarrow \operatorname{Hom}\left(F, \mathbb{C}^{*}\right) .
$$

Moreover, $\operatorname{Hom}\left(Q(R), \mathbb{C}^{*}\right)=\bar{H}$ is the maximal torus in the adjoint form $\rho(G)$ of $G$, and the induced homomorphism $H \rightarrow \bar{H} \rightarrow \operatorname{Hom}\left(\mathbb{Z}\left[W / W_{0}\right], \mathbb{C}^{*}\right)$ is just the homomorphism which sends $h \in H$ to the homomorphism $\mathbb{Z}\left[W / W_{0}\right] \rightarrow \mathbb{C}^{*}$ defined by $\alpha \mapsto \alpha(h)$. It follows that, given $a_{\alpha} \in \mathbb{C}^{*}$ for $\alpha \in R_{s}$ satisfying the conditions of the lemma, there is an $h \in H$ such that $\alpha(h)=a_{\alpha}$. Thus $\rho(h)=A$ as claimed.

\subsection{The spectral cover and the basic correspondence}

Since $\rho$ is not minuscule, the spectral cover $T$ is of the form $m_{0} \cdot \bar{T}_{1}+T_{0}$ for a positive integer $m_{0}$. The divisor $D=\bar{T}_{1} \cap T_{0}$ is nonempty, as is its preimage $\hat{D}$ in the normalization $\hat{T}_{0}$ of $T_{0}$. As the weights of $\rho$ are invariant under -1 , there is an involution $\tau$ of $\hat{T}_{0}$.

We shall need the following lemma on roots:

Lemma 5.2.1. The Weyl group $W$ acts transitively on the set of unordered triples $\{\alpha, \beta, \gamma\}$ with $\alpha, \beta, \gamma \in R_{s}$ and $\alpha+\beta+\gamma=0$. The stabilizer in $W$ of such a triple contains a cyclic permutation of the set $\{\alpha, \beta, \gamma\}$.

Proof. To prove the first statement, it suffices to prove that $W$ acts transitively on the set of unordered pairs $\{\alpha, \beta\}$ such that $\alpha, \beta$, and $\alpha+\beta$ are short roots, i.e. with the set of embeddings of a root system of type $A_{2}$ into the subroot system of short roots $G$ up to the action of the outer automorphism group of $A_{2}$. Given two such pairs $\left\{\alpha_{1}, \beta_{1}\right\}$ and $\left\{\alpha_{2}, \beta_{2}\right\}$, we may assume that $\alpha_{1}=\alpha_{2}$ since $W$ acts transitively on the set of short roots. Then $\left\{\alpha_{1}, \beta_{1}, \beta_{2}\right\}$ spans a root system of type $A_{2}$ (if $\beta_{1}=\beta_{2}$ or if the Cartan integer $n\left(\beta_{1}, \beta_{2}\right)=-1$ ) or of type $A_{3}$ (if the Cartan integer $n\left(\beta_{1}, \beta_{2}\right)$ is 0 or 1 ), and the result can be checked directly in either case.

The second statement is an immediate consequence of the fact that the Weyl group of a root system of type $A_{2}$ is the symmetric group $S_{3}$ and contains an index two subgroup as claimed.

We now define a correspondence on $\hat{T}_{0}$ which commutes with $\tau$ in an appropriate sense. As we have seen in Lemma 5.2.1, $W$ acts transitively on the set of unordered triples $\{\alpha, \beta, \gamma\}$ with $\alpha, \beta, \gamma \in R_{s}$ and $\alpha+\beta+\gamma=0$. Let $\beta \in R_{s}$ be such that $\varpi+\beta=-\gamma \in R_{s}$. (Such 
a $\beta$ exists except in case $G$ is of type $B_{n}$ or $A_{1}$.) Let $W^{\prime} \subseteq W$ be the stabilizer of the set $\{\varpi, \beta, \gamma\}$, and set $S^{\prime}=\widetilde{\mathcal{M}} / W^{\prime}$. Let $\mathcal{R}_{1}$ be the subset of ordered triples $(\alpha, \beta, \gamma) \in R_{s}^{3}$ such that $\alpha+\beta+\gamma=0$. Then $W$ acts diagonally on $\widetilde{M} \times \mathcal{R}_{1}$, and we let $S^{\prime \prime}=\left(\widetilde{M} \times \mathcal{R}_{1}\right) / W$. One can check that $S^{\prime \prime}$ is irreducible, i.e. that $W$ acts transitively on the set of ordered pairs $(\alpha, \beta)$ of short roots whose sum is a short root, as long as $G$ is not of type $A_{n}$. However, we shall not need this fact. Viewing $\hat{T}_{0}=\widetilde{\mathcal{M}} / W_{0}$ as the quotient $\left(\widetilde{\mathcal{M}} \times R_{s}\right) / W$ and similarly for $S^{\prime}$, we see that there are morphisms $S^{\prime \prime} \rightarrow \hat{T}_{0}$ and $S^{\prime \prime} \rightarrow S^{\prime}$. Thus as in the case of $E_{6}$ and $E_{7}$ there is a diagram

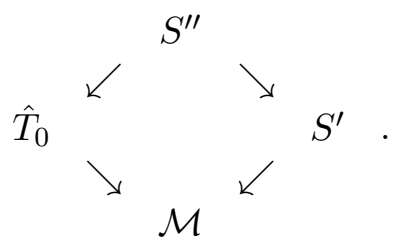

There are obvious analogues of the involution $\tau$ on $S^{\prime}$ and $S^{\prime \prime}$, for which the above morphisms are equivariant. For example, the involution on $S^{\prime \prime}$ is induced by the function $(x,(\alpha, \beta, \gamma)) \mapsto(x,(-\alpha,-\beta,-\gamma))$. We denote all of these involutions by $\tau$. Let $\nu^{\prime}: S^{\prime} \rightarrow \mathcal{M}$ and $\nu^{\prime \prime}: S^{\prime \prime} \rightarrow \mathcal{M}$ be the induced morphisms, so that $\nu^{\prime} \circ \tau=\nu^{\prime}$, and similarly for $\nu^{\prime \prime}$. We define the correspondence homomorphism $c: \hat{\nu}_{*} \mathbb{G}_{m, \hat{T}_{0}} \rightarrow \nu_{*}^{\prime} \mathbb{G}_{m, S^{\prime}}$ in the usual way, by pulling a function up to $S^{\prime \prime}$ and taking the image of the norm homomorphism from $\nu_{*}^{\prime \prime} \mathbb{G}_{m, S^{\prime \prime}}$ to $\nu_{*}^{\prime} \mathbb{G}_{m, S^{\prime}}$. Clearly, $c$ commutes with $\tau$, and so there is an induced homomorphism, which we also denote by $c$, from $\left(\hat{\nu}_{*} \mathbb{G}_{m, \hat{T}_{0}}\right)^{-}$to $\left(\nu_{*}^{\prime} \mathbb{G}_{m, S^{\prime}}\right)^{-}$.

For $G$ of type $A_{1}$ or $B_{n}$, there are no triples $\{\alpha, \beta, \gamma\}$ with $\alpha, \beta, \gamma \in R_{s}$ such that $\alpha+$ $\beta+\gamma=0$. In this case, the constructions above are vacuous and there is no correspondence to consider. In this case, we define $c$ to be the trivial homomorphism.

For future reference, we shall need the following lemma:

Lemma 5.2.2. Assume that $G$ is not of type $A_{1}$ or $B_{n}$. There is a nonempty codimension two subscheme $X$ of $S^{\prime}$ such that, for every étale open set $U \rightarrow \mathcal{M}$, if $f \in \hat{\nu}_{*} \mathcal{B}_{\hat{D}}(U)$, then $c(f) \mid U \times \mathcal{M} X=1$. If $\beta \in R_{s}$ is such that $\varpi+\beta=-\gamma \in R_{s}$ and $W^{\prime}$ is the stabilizer of the set $\{\varpi, \beta, \gamma\}$, then $X$ is the subset of $S^{\prime}=\widetilde{\mathcal{M}} / W^{\prime}$ where the functions from $S^{\prime}$ to $E_{\mathrm{reg}}$ defined by $\varpi, \beta, \gamma$ are all equal to the identity.

Proof. Let $\mathcal{R}_{1}$ be the subset of ordered triples $(\alpha, \beta, \gamma) \in R_{s}^{3}$ such that $\alpha+\beta+\gamma=0$, and let $\mathcal{R}_{2}$ be the set of unordered triples $\{\alpha, \beta, \gamma\}$ such that $\alpha, \beta, \gamma \in R_{s}$ and $\alpha+\beta+\gamma=$ 0 . There is the natural correspondence $\tilde{c}$ from $\widetilde{\mathcal{M}} \times R_{s}$ to $\widetilde{\mathcal{M}} \times \mathcal{R}_{2}$ defined as follows. Let $\varphi_{1}: \widetilde{\mathcal{M}} \times \mathcal{R}_{1} \rightarrow \widetilde{\mathcal{M}} \times R_{s}$ and $\varphi_{2}: \widetilde{\mathcal{M}} \times \mathcal{R}_{1} \rightarrow \widetilde{\mathcal{M}} \times \mathcal{R}_{2}$ be the obvious morphisms: $\varphi_{1}(x,(\alpha, \beta, \gamma))=(x, \alpha)$ and $\varphi_{2}(x,(\alpha, \beta, \gamma))=(x,\{\alpha, \beta, \gamma\})$. Then $\tilde{c}(g)$ is the pullback of $g$ under $\varphi_{1}$, followed by the norm map induced by $\varphi_{2}$. Explicitly,

$$
\tilde{c}(g)(x,\{\alpha, \beta, \gamma\})=g(x, \alpha) g(x, \beta) g(x, \gamma) .
$$

The function $g$ is the pullback of a function $f$ on $\hat{T}_{0}=\left(\widetilde{\mathcal{M}} \times R_{s}\right) / W$, if and only if it is 
$W$-invariant. In this case, $\tilde{c}(g)$ is also $W$-invariant, and hence is the pullback of a function on $S^{\prime}$, which is clearly $c(f)$.

Let $f$ be a function on $\hat{T}_{0}$ which is 1 on $\hat{D}$. Then its pullback $g$ to $\widetilde{\mathcal{M}} \times R_{s}$ is identically 1 on $\coprod_{\alpha \in R_{s}} D_{\alpha}$, where

$$
D_{\alpha}=\left\{(x, \alpha) \in \widetilde{\mathcal{M}} \times R_{s}: \alpha(x) \text { is the identity } p_{0}\right\} .
$$

Thus $\tilde{c}(g)$ is identically 1 on the subscheme $\coprod_{\{\alpha, \beta, \gamma\} \in \mathcal{R}_{2}} X_{\alpha, \beta, \gamma}$, where

$$
X_{\alpha, \beta, \gamma}=\left\{(x,\{\alpha, \beta, \gamma\}) \in \widetilde{\mathcal{M}} \times \mathcal{R}_{2}: \alpha(x)=\beta(x)=\gamma(x) \text { is the identity } p_{0}\right\} .
$$

Clearly $X_{\alpha, \beta, \gamma}$ is nonempty and $W$-invariant, and it is of codimension two since $\alpha+\beta+\gamma=0$. Hence $\coprod_{\{\alpha, \beta, \gamma\} \in \mathcal{R}_{2}} X_{\alpha, \beta, \gamma}$ is the pullback of a nonempty codimension two subset $X$ of $S^{\prime}$ which has the required properties.

We now claim:

Proposition 5.2.3. The sheaf $\overline{\mathcal{A}}_{\rho}$ is isomorphic to the closed subsheaf $\operatorname{Ker} c \cap\left(\hat{\nu}_{*} \mathcal{B}_{\hat{D}}\right)^{-}$of $\left(\hat{\nu}_{*} \mathcal{B}_{\hat{D}}\right)^{-}$.

Proof. By Corollary 3.4.6, $\overline{\mathcal{A}}_{\rho}$ is isomorphic to a closed subsheaf of $\hat{\nu}_{*} \mathcal{B}_{\hat{D}}$. Thus it suffices to prove that, via this isomorphism, $\overline{\mathcal{A}}_{\rho} \cong \hat{\nu}_{*} \mathcal{B}_{\hat{D}} \cap\left(\hat{\nu}_{*} \mathbb{G}_{m, \hat{T}_{0}}\right)^{-} \cap \operatorname{Ker} c$. Since both the image of $\overline{\mathcal{A}}_{\rho}$ and $\hat{\nu}_{*} \mathcal{B}_{\hat{D}} \cap\left(\hat{\nu}_{*} \mathbb{G}_{m, \hat{T}_{0}}\right)^{-} \cap \operatorname{Ker} c$ are closed in $\hat{\nu}_{*} \mathcal{B}_{\hat{D}}$, it suffices to prove that their restrictions to an open dense set agree. As usual, we use the open set $U \rightarrow \mathcal{M}$ of Lemma 3.1.2 and Corollary 3.1.3, and we shall prove that their restrictions to $U$ agree. Note that $\hat{\nu}(\hat{D})$ does not lie in the image of $U$. Over $U, \mathcal{A}$ is the sheaf of sections of the constant group scheme $U \times H$ and hence $\overline{\mathcal{A}}_{\rho}$ is the sheaf of sections of the constant group scheme $U \times \bar{H}$. The fiber of $\hat{T}_{0}$ over a point $x$ in the image of $U$ is indexed by the weights of $\rho$, i.e. by $R_{s}$. Thus if $f \in \hat{\nu}_{*} \mathbb{G}_{m, \hat{T}_{0}}(U)$, its value at $x$ is given by elements $a_{\alpha} \in \mathbb{C}^{*}$. To say that $f \in\left(\hat{\nu}_{*} \mathbb{G}_{m, \hat{T}_{0}}\right)^{-} \cap \operatorname{Ker} c$ means exactly that $a_{\alpha} a_{-\alpha}=1$ and that, for every triple $(\alpha, \beta, \gamma) \in R_{s}^{3}$ such that $\alpha+\beta+\gamma=0, a_{\alpha} a_{\beta} a_{\gamma}=1$. By Lemma 5.1.1, this is exactly the condition that $f$ lies in the image of $\overline{\mathcal{A}}_{\rho}$.

Proof of Theorem 2.2.3. By Lemma 3.1.4, to prove Theorem 2.2.3, it will suffice to prove that $H^{1}\left(\mathcal{M} ; \overline{\mathcal{A}}_{\rho}\right)=\{1\}$ and that $H^{0}\left(\mathcal{M} ; \overline{\mathcal{A}}_{\rho}\right)=\{1\}$ or $\mathbb{Z}$ depending on whether $E$ is cuspidal or nodal. If $c$ is trivial, then $\overline{\mathcal{A}}_{\rho}=\left(\hat{\nu}_{*} \mathcal{B}_{\hat{D}}\right)^{-}$and the result is just Lemma 3.5.4. So we may assume that $c$ is not trivial. Restricting $c$ to $\left(\hat{\nu}_{*} \mathcal{B}_{\hat{D}}\right)^{-}$and applying Proposition 5.2.3 gives an exact sequence

$$
\{1\} \rightarrow \overline{\mathcal{A}}_{\rho} \rightarrow\left(\hat{\nu}_{*} \mathcal{B}_{\hat{D}}\right)^{-} \rightarrow \mathcal{I} \rightarrow\{1\}
$$

where $\mathcal{I}$ is the image of $\left(\hat{\nu}_{*} \mathcal{B}_{\hat{D}}\right)^{-}$in $\nu_{*}^{\prime} \mathbb{G}_{m, S^{\prime}}$ 
As usual, assume first that $E$ is cuspidal. Since $S^{\prime}$ is the quotient of the affine space $\mathfrak{h}$ by a finite group, $H^{0}\left(\mathcal{M} ; \nu_{*}^{\prime} \mathbb{G}_{m, S^{\prime}}\right) \cong \mathbb{C}^{*}$ is identified with the group of nonzero constant functions on $S^{\prime}$. Since $H^{0}\left(\mathcal{M} ;\left(\hat{\nu}_{*} \mathcal{B}_{\hat{D}}\right)^{-}\right)=H^{1}\left(\mathcal{M} ;\left(\hat{\nu}_{*} \mathcal{B}_{\hat{D}}\right)^{-}\right)=\{1\}$ in this case, by Lemma 3.5.4, it suffices to show that $H^{0}(\mathcal{M} ; \mathcal{I})=\{1\}$. In any case, $H^{0}(\mathcal{M} ; \mathcal{I})$ is a subsheaf of $H^{0}\left(\mathcal{M} ; \nu_{*}^{\prime} \mathbb{G}_{m, S^{\prime}}\right) \cong \mathbb{C}^{*}$ and hence consists of constant functions on $S^{\prime}$. To determine the value of these constant functions, it suffices to restrict to the nonempty subset $X$ of Lemma 5.2.2, where we have seen that the image of any local section of $\hat{\nu}_{*} \mathcal{B}_{\hat{D}}$ takes the value 1 . Hence $H^{0}(\mathcal{M} ; \mathcal{I})=\{1\}$, concluding the proof in the cuspidal case.

Finally assume that $E$ is nodal. In this case, by Lemma 3.5.4, $H^{0}\left(\mathcal{M} ;\left(\hat{\nu}_{*} \mathcal{B}_{\hat{D}}\right)^{-}\right) \cong \mathbb{Z}$ and $H^{1}\left(\mathcal{M} ;\left(\hat{\nu}_{*} \mathcal{B}_{\hat{D}}\right)^{-}\right)=\{1\}$. We must again show that $H^{0}(\mathcal{M} ; \mathcal{I})=\{1\}$. It suffices to show that

$$
\left\{g \in H^{0}\left(S^{\prime} ; \mathbb{G}_{m, S^{\prime}}\right): g \mid X=1\right\}=\{1\} .
$$

We have seen that $S^{\prime}=H / W^{\prime}$, where $W^{\prime}$ is the stabilizer of an unordered triple of short roots $\{\varpi, \beta, \gamma\}$ whose sum is zero. Thus

$$
H^{0}\left(S^{\prime} ; \mathbb{G}_{m, S^{\prime}}\right) \subseteq\left(H^{0}\left(H ; \mathbb{G}_{m, H}\right)\right)^{W^{\prime}}=\left(\mathbb{C}^{*} \times \mathbf{X}(H)\right)^{W^{\prime}}=\mathbb{C}^{*} \times \mathbf{X}(H)^{W^{\prime}} .
$$

Suppose that $g \in H^{0}\left(S^{\prime} ; \mathbb{G}_{m, S^{\prime}}\right)$ and $g \mid X=1$. Then, since the origin 1 of $H$ is contained in the pullback of $X$, the pullback of $g$ is the identity at $1 \in H$. It follows that the pullback of $g$ lies in $\mathrm{X}(H)^{W^{\prime}}$.

By Lemma 5.2.2, the preimage of $X$ in $H$ contains the identity component of $\operatorname{Ker} \varpi \cap$ $\operatorname{Ker} \beta \cap \operatorname{Ker} \gamma$, which is a subtorus $H^{\prime}$ of codimension two. In particular, the pullback of $g$ is equal to 1 on $H^{\prime}$. Let $H^{\prime \prime}$ be the subtorus of $H$ whose Lie algebra is spanned by the coroots $\varpi^{\vee}, \beta^{\vee}$, and $\gamma^{\vee}$. The induced homomorphism $H^{\prime} \times H^{\prime \prime} \rightarrow H$ is a $W^{\prime}$-equivariant isogeny, so that

$$
\mathrm{X}(H)^{W^{\prime}} \subseteq \mathrm{X}\left(H^{\prime}\right)^{W^{\prime}} \oplus \mathrm{X}\left(H^{\prime \prime}\right)^{W^{\prime}} .
$$

Since $g$ has trivial restriction to $H^{\prime}$, the image of $g$ lies in $\mathrm{X}\left(H^{\prime \prime}\right)^{W^{\prime}}$. Here $\mathrm{X}\left(H^{\prime \prime}\right) \cong \mathbb{Z}^{2}$ is the weight lattice of a root system of type $A_{2}$, and $W^{\prime}$ contains a subgroup of order 3 acting on $\mathbb{Z}^{2}$ in the standard way. It follows that $\mathrm{X}\left(H^{\prime \prime}\right)^{W^{\prime}}=0$, so that $H^{0}(\mathcal{M} ; \mathcal{I})=\{1\}$ as claimed.

This completes the proof of Theorem 2.2.3.

\section{References}

[1] J. F. Adams, Lectures on Exceptional Lie Groups, University of Chicago Press, Chicago, 1996.

[2] N. Bourbaki, Groupes et Algèbres de Lie, Chap. 4, 5, et 6, Masson, Paris, 1981.

[3] P. Deligne, Cohomologie Etale: Séminaire de Géométrie Algébrique du Bois-Marie $S G A 4 \frac{1}{2}$, Lecture Notes in Mathematics 569, Springer, Berlin/Heidelberg/New York, 1977. 
[4] R. Donagi, Spectral covers, in Current Topics in Complex Algebraic Geometry (Berkeley 1992/93), MSRI Publications 28, Cambridge University Press, Cambridge , 1995.

[5] R. Donagi and D. Gaitsgory, The gerbe of Higgs bundles, Transform. Groups 7 (2002), $109-153$.

[6] E. Freitag and R. Kiehl, Etale Cohomology and the Weil Conjecture, Ergebnisse der Mathematik und ihrer Grenzgebiete 3. Folge 13, Springer, Berlin Heidelberg, 1988.

[7] R. Friedman and J.W. Morgan, Holomorphic principal bundles over elliptic curves, math.AG/9811130.

[8] R. Friedman and J.W. Morgan, Holomorphic principal bundles over elliptic curves II: The parabolic construction, J. Differential Geometry 56 (2001), 301-379, math.AG/0006174.

[9] R. Friedman and J.W. Morgan, Holomorphic principal bundles over elliptic curves III: Singular curves and fibrations, math.AG/0108104.

[10] R. Friedman and J.W. Morgan, Minuscule representations, invariant polynomials, and spectral covers, math.AG/0011082.

[11] R. Friedman, J.W. Morgan, and E. Witten, Vector bundles over elliptic fibrations, J. Algebraic Geometry 8 (1999), 279-401.

[12] V. Kanev, Spectral covers and Prym-Tjurin varieties I, in Abelian Varieties, Proceedings of the International Conference (Egloffstein 1993), W. Barth, K. Hulek, and H. Lange eds., de Gruyter, Berlin-New York, 1995.

[13] F. Knop, Automorphisms, root systems, and compactifications of homogeneous varieties, Jour. Amer. Math. Soc. 9 (1996), 153-174.

[14] B. Kostant, Lie group representations on polynomial rings, Am. J. Math. 85 (1963), 327-404.

[15] R. Steinberg, Regular elements of semisimple algebraic groups, Publ. Math. Inst. Hautes Études Sci. 25 (1965), 49-80. 